\title{
Gravitational corrections to Higgs potentials
}

\section{Marios Bounakis and Ian G. Moss}

School of Mathematics, Statistics and Physics, Newcastle University, Newcastle Upon Tyne, NE1 7RU, U.K.

E-mail: M.Bounakis2@newcastle.ac.uk, ian.moss@ncl.ac.uk

ABSTRACT: Understanding the Higgs potential at large field values corresponding to scales in the range above $10^{10} \mathrm{GeV}$ is important for questions of vacuum stability, particularly in the early universe where survival of the Higgs vacuum can be an issue. In this paper we show that the Higgs potential can be derived in away which is independent of the choice of conformal frame for the spacetime metric. Questions about vacuum stability can therefore be answered unambiguously. We show that frame independence leads to new relations between the beta functions of the theory and we give improved limits on the allowed values of the Higgs curvature coupling for stability.

Keywords: Cosmology of Theories beyond the SM, Effective Field Theories

ARXIV EPRINT: 1710.02987 


\section{Contents}

1 Introduction 1

2 Covariant effective actions 5

3 The gravity-Higgs effective field theory 10

4 Expansions of the gravity-Higgs action $\quad 11$

$\begin{array}{lll}4.1 & \text { First order variations } & 12\end{array}$

$\begin{array}{lll}4.2 & \text { Second order variations } & 12\end{array}$

$\begin{array}{lll}4.3 & \text { Vilkovisky-DeWitt corrections } & 14\end{array}$

5 Gravity-Higgs mode expansions $\quad 14$

6 Gauge bosons, Goldstone modes and fermions 18

$\begin{array}{lll}7 & \text { The effective potential and stability } & 21\end{array}$

8 Conclusion $\quad 25$

$\begin{array}{ll}\text { A Mode decomposition matrices } & 26\end{array}$

$\begin{array}{ll}\text { B Zeta-function evaluation } & 27\end{array}$

\section{Introduction}

Extrapolation of the Standard Model of particle physics to high energies leads to the remarkable conclusion that our vacuum is only a long-lived metastable state, in which the Higgs field sits at a local minimum of the Higgs potential surrounded by a potential barrier of width somewhere in the range $10^{10}-10^{14} \mathrm{GeV}[1-3]$. This raises an interesting question about initial conditions, because, if the Standard Model is correct at these energies, then somehow the Higgs field had to evolve into the metastable vacuum state during the early stages of the universe [4].

The Higgs potential barrier depends strongly on the effective Higgs mass at high energies, and it is quite possible that gravitational corrections may be important. In the relevant energy range, there is no reason to abandon General Relativity as 'an effective field theory' description of gravity [5]. There are two contributions to the effective Higgs mass, the ordinary one and the mass due to the coupling $\xi R \mathcal{H}^{\dagger} \mathcal{H}$, between the Higgs field $\mathcal{H}$ and the curvature $R$. We will assume that inflation is driven by an inflaton field, not the Higgs field, which is assumed weakly interacting and makes no contribution to the Higgs potential. The curvature coupling increases the height of the potential barrier around the 
metastable minimum if $\xi R$ is positive, and has the opposite effect when $\xi R$ is negative, making Higgs stability sensitive to the value of $\xi$.

Placing the Higgs decay into a cosmological context introduces an ambiguity in how we define the spacetime geometry. In particular, we can perform a conformal re-scaling of the metric which removes the curvature-coupling term, transforming the theory from the original Jordan frame metric to the Einstein frame metric. It has been noted that quantum calculations can sometimes lead to different results when done in the Jordan or the Einstein frame $[6,7]$. This is a puzzle, because we want to avoid a situation in which the Higgs field is unstable in the Jordan frame and stable in the Einstein frame. The contradiction would be best resolved by having an approach to quantisation which is consistent whatever the choice of spacetime metric [8-11]. We shall show that there is a covariant quantisation scheme which gives consistent results on Higgs instability.

The basic tool we use is an effective action which is covariant under field transformations [12-15]. This is a stronger requirement than General Covariance, or covariance under spacetime coordinate transformations. The idea of a field-space covariant quantum field theory (hereafter called covariant) is illustrated by the diagram in eq. (1.1). Quantisation followed by a field redefinition should give the same result as starting from a field redefinition and then quantising, i.e. the diagram should commute.

$$
\begin{array}{ccc}
\varphi & \rightarrow & \varphi^{\prime} \\
\downarrow & & \downarrow \\
\Gamma[\varphi] & \rightarrow & \Gamma\left[\varphi^{\prime}\right]
\end{array}
$$

Demanding covariance of the effective action guarantees covariance of the effective field equations. Without covariance, there is a different quantum field theory for each choice of field variables. Imposing covariance has another virtue. Terms are added to the classical Lagrangian to fix the gauge freedom. Solutions to the usual effective field equations depend on the choice of these gauge-fixing terms. However, in the covariant approach, the solutions to the effective field equations are independent of the gauge-fixing terms.

Covariant approaches are widely used to quantise non-linear sigma models [16, 17], but they are very rarely used for gauge theories. One reason they are not widely used is that the gauge-fixing dependence of the usual effective action is not considered problematic, since the dependence goes away 'on-shell' , i.e. the action takes the same value at solutions to the effective field equations [18-21]. Furthermore, it is easy to show that the Jordan and Einstein frame Higgs theories have equivalent perturbative expansions when the background fields are on-shell and the Higgs field is small [22].

Using a covariant approach retains the gauge-fixing and frame independence off-shell, for any range of Higgs field. On the other hand, covariant approaches are not totally unambiguous, because there are two versions of the covariant effective action: $\Gamma\left[\varphi^{*}, \varphi\right]$ which generates the 1PI diagrams but depends on an extra field $\varphi^{*}$ [23], and the DeWitt effective action $\Gamma[\varphi]$ which does not generate 1PI diagrams [24]. Fortunately, both generate the same effective field equations, and they agree on-shell. They are therefore equivalent for questions of vacuum stability, so we will use the simpler DeWitt effective action. 
Higgs vacuum decay is a situation where the effective action and the classical action lead to very different qualitative behaviour [25-28]. Another example is Coleman-Weinberg theory of massless electrodynamics, where quantum corrections to the effective action lead to symmetry breaking. In these situations, we use solutions to the renormalisation group corrected field equations with running coupling constants to determine the vacuum state or to calculate tunnelling amplitudes [29]. Note that we use 'on shell' to refer to fields which satisfy the effective field equations rather than the classical field equations. We will investigate whether covariant and non-covariant approaches to the effective action give different physical results by doing specific calculations of the running couplings in the Higgs effective potential.

The renormalisation group corrected potential used here is constructed as follows. The DeWitt effective action for the modulus of the Higgs field $\phi$ is written as a functional $\Gamma\left(g_{i}, \phi, g_{\mu \nu}, \mu_{R}\right)$, where $g_{i}$ are running couplings depending on $\mu_{R}$, the renormalisation scale. At one loop order, the explicit dependence on renormalisation scale has contributions from all types of field in the standard model. These contributions are determined by perturbation theory and depend on a set of second order differential operators $\Delta^{n}(\phi)$. Following Coleman and Weinberg [29], the $\beta$ functions can be obtained by comparing coefficients in the renormalisation group equation for the Lagrangian,

$$
\sum_{i} \beta_{i} \frac{\partial \mathcal{L}}{\partial g_{i}}-\gamma_{\phi} \phi \frac{\partial \mathcal{L}}{\partial \phi}-\gamma_{g} g_{\mu \nu} \frac{\partial \mathcal{L}}{\partial g_{\mu \nu}}=\frac{1}{16 \pi^{2}} \sum_{n}( \pm) b_{2}\left(\Delta^{n}\right)
$$

where the sign is positive for bosons and negative for fermions and ghosts. Renormalisation of the fields is responsible for the anomalous dimensions $\gamma_{\phi}$ and $\gamma_{g}$ (where we are using the sign conventions of [28]). The functions $b_{2}$ are polynomial combinations of coefficients in the operators $\Delta^{n}$. General expressions for $b_{2}$ are known for many types of operators on arbitrary spacetime backgrounds (e.g. [30]). Since the theory we are dealing with is not renormalisable, the Lagrangian has an infinite series of terms which has to be truncated at some inverse power of the the cutoff scale of the theory, which we naturally take to be the Planck mass. At one loop, the $b_{2}$ coefficient gives us terms up to order $M_{p}^{-4}$.

A change of variable from $\mu_{R}$ to $t=\ln \left(\phi / \mu_{R}\right)$ changes the functional form of the couplings in the effective action from $g_{i}\left(\mu_{R}\right)$ to $g_{i}^{\prime}(t)$,

$$
\Gamma^{\prime}\left(g_{i}^{\prime}(t), \phi^{\prime}(t), g_{\mu \nu}^{\prime}(t), t\right)=\Gamma\left(g_{i}\left(\mu_{R}\right), \phi\left(\mu_{R}\right), g_{\mu \nu}\left(\mu_{R}\right), \mu_{R}\right)
$$

The renormalisation group corrected Lagrangian is defined by the leading term, $\mathcal{L}^{\prime}\left(g_{i}^{\prime}(t), \phi^{\prime}(t), g_{\mu \nu}^{\prime}(t)\right)$. The dependence of the parameters on the Higgs field on $\phi$ is determined by the renormalisation group, which implies

$$
\frac{d g_{i}^{\prime}}{d t}=\frac{\beta_{i}\left(g_{j}^{\prime}\right)}{1+\gamma_{\phi}\left(g_{j}^{\prime}\right)},
$$

subject to values fixed at a given (low energy) mass scale $M$.

The first thing to note about the Coleman-Weinberg method for calculating the $\beta$ functions is that it relies on the functional form of the effective action. Therefore a knowledge 
of the effective action which is only valid for solutions to the background field equations is not sufficient. The covariant effective action gives us an unambiguous off-shell formulation and a unique set of beta functions. In order to construct this covariant effective action we make use of the non-trivial geometry of the space of metrics and fields. In the general case of a gauge theory with fields $\varphi^{I}$ and action $S[\varphi]$, the covariant operator $\Delta_{I J}$ for the field fluctuations is given by $[12,13]$

$$
\Delta_{I J}=-\frac{\delta^{2} S}{\delta \varphi^{I} \delta \varphi^{J}}+\Gamma^{K}{ }_{I J} \frac{\delta S}{\delta \varphi^{K}}+\lambda_{g} R^{I \alpha}[\varphi] R_{I \alpha}[\varphi],
$$

The innovation of Vilkovisky and DeWitt was to put the second functional derivatives into covariant form by introducing a field-space connection $\nabla_{I}$ with connection coefficients $\Gamma^{K}{ }_{I J}$. The connection ensures that the effective action is covariant under field redefinitions. In the Landau gauge limit $\lambda_{g} \rightarrow \infty$, the connection coefficients reduce to the Levi-Civita connection coefficients for the local metric on the space of fields. The final term in (1.5) is a gauge-fixing term. Details of the covariant approach are given in section 2 .

The connection term vanishes when the background field satisfies the classical field equations i.e. $\delta S / \delta \varphi^{I}=0$, and then non-covariant and covariant effective actions agree. However, we might expect differing results when the background satisfies the quantum corrected field equations. The beta-functions and the renormalisation group corrected effective Lagrangians defined using non-covariant and covariant approaches need not be the same.

In sections 4 and 5 we will calculate the Higgs parameter beta-functions in both covariant and non-covariant form in the Einstein and Jordan frames. As is well known, the beta-function for the curvature coupling $\beta_{\xi} \propto 6 \xi-1$ in the Jordan frame. This standard result cannot hold in a covariant approach, because $\xi$ vanishes in the Einstein frame and therefore the covariant $\beta_{\xi}$ cannot depend on $\xi .{ }^{1}$ As expected, when we do the calculation, the non-covariant results are frame dependent whilst the covariant results are frame independent. However, the combination $\mu^{2}+\xi R$, which acts as an effective Higgs mass, and the Higgs self-coupling $\lambda$ have the same scale behaviour in non-covariant and covariant approaches. The leading behaviour of the renormalisation group effective potential is therefore frame independent, and differences arise only in terms that are suppressed by factors of $M_{p}^{-4}$. Our results for stability with the renormalisation group corrected potential are therefore similar to those found previously [31].

In section 4 we will explore some of the consequences of the covariant approach. One of these is that field redefinitions mix some of the parameters of the theory, and having covariance leads to a set of relations between the beta functions for these parameters. These relations can be used, for example, to completely determine the dependence of the betafunctions on the curvature coupling $\xi$. Another consequence of using a covariant approach is that the path integral is independent of the gauge-fixing terms in the Lagrangian. Therefore quantum tunnelling rates will be unambiguous. In non-covariant approaches, this issue is non-trivial, and independence has only been demonstrated explicitly when the true vacuum is not radiatively generated [32, 33].

\footnotetext{
${ }^{1}$ In fact, $\xi$ appears as a correction to the mass in the Einstein frame, and contributes to $\beta_{\mu^{2}}$.
} 
In section 7, we look at a practical application of our results to the decay of the Higgs vacuum during inflation. The running couplings make a considerable difference to the Higgs decay rates when the curvature coupling is small. This is consistent with earlier work by Herranen et al. [31], but we give more precise results and we confirm that the results are the same in both the Jordan and Einstein frame. We also find a regime in which the Higgs potential has two maxima. We only consider vacuum decay using tunnelling with the simplest type of instanton, thought recent work has shown the existence of more complicated instanton solutions [34].

This paper focuses on the UV behaviour of the quantum theory and how this affects the Higgs potential in de Sitter space. In many ways, though, the IR behaviour of Higgs fields in de Sitter space is a more interesting subject. It has become apparent, initially from stochastic theory [35-38] and also from infra-red expansions [39, 40], that a selfcoupled massless scalar field in a de Sitter invariant state acquires a mass squared of order $\lambda^{1 / 2} H^{2}$, where $H$ is the expansion rate. This limits the applicability of our results for small curvature coupling. It also means that, when integrating the renormalisation group equations for the effective mass in de Sitter space, we start with this IR mass, rather than the low energy Higgs mass.

\section{Covariant effective actions}

The aim of this section is to introduce the field-covariant effective action and to give two methods for evaluating the action to one loop order, specifically by taking the Landau gauge-fixing limit and by decomposition into gauge-fixed and pure gauge modes, leading to the results quoted in the introduction. Field components are denoted by indices $I, J, \ldots$ and gauge parameters by indices $\alpha, \beta, \ldots$ Condensed notation is used throughout, with contractions over $I, J, \ldots$ denoting integration over spacetime and functional derivatives with respect to the fields denoted by $\partial_{I}$.

A field-covariant effective action can be constructed whenever there exists a covariant notion of the distance between two field configurations. Formally, this means we have a Riemannian geometry on the space of fields $\varphi^{I}$ and geodesics can be defined $[12,13,15]$. This geometry allows us to replace an ordinary field displacement $\varphi^{I}-\phi^{I}$ with the covariant tangent vector to the geodesic from $\phi^{I}$ to $\varphi^{I}$, which we denote by $\sigma^{I}(\phi, \varphi)$. The metric can also be used to define a field-space invariant volume measure $D \varphi$ for functional integration.

Our starting point is the covariant action of Burgess and Kunstatter [15, 23], defined implicitly by,

$$
e^{i \Gamma\left[\phi, \phi_{*}\right]}=\int D \varphi e^{i S[\varphi]-i\left(\delta \Gamma / \delta \sigma^{J}\right)\left(\sigma^{J}\left[\phi_{*}, \phi\right]-\sigma^{J}\left[\phi_{*}, \varphi\right]\right)} .
$$

This depends on the effective field $\phi^{I}$ and an arbitrary expansion point $\phi_{*}^{I}$. The effective action generates effective field equations for $\phi^{I}$, in the the sense that

$$
\partial_{I} \Gamma\left[\phi, \phi_{*}\right]=0 \Longrightarrow\langle\sigma[\phi, \hat{\varphi}]\rangle=0,
$$

where $\sigma[\phi, \hat{\varphi}]$ is the geodesic distance and $\hat{\varphi}^{I}$ is the field operator. In the covariant approach, $\phi^{I} \neq\left\langle\hat{\varphi}^{I}\right\rangle$, but instead $\phi^{I}$ is the classical field which is closest to the quantum field using the 
invariant distance. Note that the effective field equations do not depend on the expansion point $\phi_{*}$. We make use of this fact and choose $\phi_{*}^{I}=\phi^{I}$, which defines the DeWitt effective action $[15,24]$,

$$
\Gamma[\phi]=\Gamma[\phi, \phi]
$$

The DeWitt effective action generates the effective field equations using $\partial_{I} \Gamma[\phi]=0$.

In a gauge theory, there are infinitesimal gauge transformations of the field $\varphi^{I}$ of the form

$$
\delta \varphi^{I}=R_{\alpha}^{I} \epsilon^{\alpha},
$$

which leave the action invariant, i.e. $R^{I}{ }_{\alpha} \partial_{I} S=0$. The gauge is fixed using a gauge-fixing functional $\chi^{\alpha}[\phi, \varphi]$, and then the path integral is modified as follows,

$$
S[\varphi] \rightarrow S[\varphi]+\frac{1}{2} \gamma_{\alpha \beta} \chi^{\alpha} \chi^{\beta}+\frac{\hbar}{i} \operatorname{tr} \ln Q_{\beta}^{\alpha},
$$

This introduces a metric $\gamma_{\alpha \beta}$ on the gauge parameters and a ghost operator

$$
Q_{\beta}^{\alpha}=\left(\partial_{I} \chi^{\alpha}\right) R_{\beta}^{I}
$$

Next, a procedure developed by Vilkovisky and DeWitt [12] generates the geometry on field space which guarantees that the effective action is:

1. Covariant under field redefinitions of $\varphi^{I}$;

2. Independent of the choice of gauge fixing functional $\chi^{\alpha}$;

3. Independent of the metric $\gamma_{\alpha \beta}$.

The field-space geometry includes a local field-space metric $\mathcal{G}_{I J}$ and a non-local field space connection $\nabla_{I}$. The metric allows an orthogonal decomposition of field variations into pure gauge and gauge-fixed directions. Projection in the pure-gauge direction can be done using

$$
\bar{\Pi}_{J}^{I}=R_{\alpha}^{I} \mathcal{N}^{\alpha \beta} R_{J \beta},
$$

where indices are lowered using the metric tensors in the usual way, and the normalisation factor appearing here is

$$
\mathcal{N}^{\alpha \beta}=\left(R_{\alpha}^{I} R_{I \beta}\right)^{-1}
$$

When this is applied to a gauge variation, $\bar{\Pi}_{J}^{I} \delta \varphi^{J}=\delta \varphi^{I}$. The orthogonal projection in the gauge-fixed direction is the DeWitt projection $\Pi=\mathbb{I}-\bar{\Pi}$.

The local metric also generates a Levy-Civita connection on field space, denoted by $\mathcal{D}_{I}$, for example

$$
\mathcal{D}_{I} \mathcal{D}_{J} S=\partial_{I} \partial_{J} S-\Gamma^{K}{ }_{I J} \partial_{K} S .
$$

In a gauge theory, the Vilkovisky-DeWitt connection $\nabla_{I}$ is not equal to the Levy-Civita connection, but it is related to it by the projection operators,

$$
\nabla^{I} \nabla_{J} S=\Pi\left(\mathcal{D}^{I} \mathcal{D}_{J} S\right) \Pi .
$$


One of the disadvantages of using a covariant approach is that this expression is non-local. However, we will now describe ways to deal with this non-locality at one loop.

At one-loop, the contribution to the covariant effective action obtained from a geodesic expansion of the fields in the path integral is

$$
\Gamma^{(1)}=\frac{\hbar}{2 i} \operatorname{tr} \ln \left\{\Pi\left(\mathcal{D}^{I} \mathcal{D}_{J} S\right) \Pi+\left(\partial^{I} \chi^{\alpha}\right)\left(\partial_{J} \chi_{\alpha}\right)\right\}-\frac{\hbar}{i} \operatorname{tr} \ln Q_{\beta}^{\alpha},
$$

For an actual calculation, we can reduce the amount of work by choosing a convenient gauge-fixing functional, in particular the $\mathcal{R}_{\xi}$ gauges in which $\partial_{I} \chi^{\alpha}=\lambda_{g}^{1 / 2} R_{I}^{\alpha}$, where $\lambda_{g}$ is a constant gauge-fixing parameter. The one-loop effective action is then

$$
\Gamma^{(1)}=\frac{\hbar}{2 i} \operatorname{tr} \ln \left\{\Pi\left(\mathcal{D}^{I} \mathcal{D}_{J} S\right) \Pi+\lambda_{g} R^{I \alpha} R_{J \alpha}\right\}-\frac{\hbar}{i} \operatorname{tr} \ln \left\{\lambda_{g}^{1 / 2} R^{I \alpha} R_{I \beta}\right\} .
$$

If the covariant derivatives in (2.9) are replaced by ordinary functional derivatives, and the projections are dropped, then the result is a non-covariant effective action contribution $\Gamma_{\mathrm{nc}}^{(1)}$,

$$
\Gamma_{\mathrm{nc}}^{(1)}=\frac{\hbar}{2 i} \operatorname{tr} \ln \left\{\partial^{I} \partial_{J} S+\lambda_{g} R^{I \alpha} R_{J \alpha}\right\}-\frac{\hbar}{i} \operatorname{tr} \ln \left\{\lambda_{g}^{1 / 2} R^{I \alpha} R_{I \beta}\right\} .
$$

If the background fields are 'on shell', specifically when $\partial_{I} S=0$, then the connection $\nabla_{I} \rightarrow$ $\partial_{I}$, and the covariant and non-covariant results coincide, $\Gamma^{(1)}=\Gamma_{\mathrm{nc}}^{(1)}$. Most calculations are done on shell, and eq. (2.13) is the traditional route to evaluation of the effective action.

We will show that the off-shell result can be simplified in two equivalent ways. Firstly

$$
\Gamma^{(1)}=\frac{\hbar}{2 i} \operatorname{tr} \ln \left\{\Pi\left(\mathcal{D}^{I} \mathcal{D}_{J} S\right) \Pi\right\}-\frac{\hbar}{2 i} \operatorname{tr} \ln \left\{R^{I \alpha} R_{I \beta}\right\},
$$

where the logarithms are interpreted in a particular way described below. If we have $n$ fields and $m$ gauge variations, then there are $n-m$ non-gauge fields but there are $n-2 m$ degrees of freedom. The ghost contribution accounts for the difference between these two.

The second method is to use the Landau gauge $\lambda_{g} \rightarrow \infty$,

$$
\Gamma^{(1)}=\lim _{\lambda_{g} \rightarrow \infty} \frac{\hbar}{2 i} \operatorname{tr} \ln \left\{\mathcal{D}^{I} \mathcal{D}_{J} S+\lambda_{g} R^{I \alpha} R_{J \alpha}\right\}-\frac{\hbar}{i} \operatorname{tr} \ln \left\{\lambda_{g}^{1 / 2} R^{I \alpha} R_{I \beta}\right\},
$$

This appears to be more complicated, but the advantage of this method is that removing the projection operators leaves an operator which is explicitly local in spacetime, making it suitable for adiabatic expansion techniques.

For simplicity, we define the functional traces using Euclidean methods with

$$
\operatorname{tr} \ln A_{L}=-i \zeta^{\prime}(0, A)-i \zeta(0, A) \ln \mu_{R}^{2},
$$

where $A$ is a positive definite operator obtained from the Lorentzian operator $A_{L}$ by analytic continuation of the time-like coordinate. This limits us to metrics with a valid analytic continuation. The generalised zeta-function is defined by $\zeta(s, A)=\operatorname{tr} A^{-s}$ and $\mu_{R}$ is the renormalisation scale. We can read off the scaling of the Euclidean effective action $\Gamma_{E}$ from (2.15)

$$
\mu_{R} \frac{d \Gamma_{E}^{(1)}}{d \mu_{R}}=\hbar \lim _{\lambda_{g} \rightarrow \infty}\left\{-\zeta\left(0, \mathcal{D}^{I} \mathcal{D}_{J} S+\lambda_{g} R^{I \alpha} R_{J \alpha}\right)+2 \zeta\left(0, \lambda_{g}^{1 / 2} \mathcal{R}^{I \alpha} \mathcal{R}_{I \beta}\right)\right\}
$$


In Landau gauge, the operators are local, and it is possible to prove that $\zeta(0, A)$ can be expressed in terms of a local adiabatic expansion coefficient $b_{2}(A)$,

$$
\zeta(0, A)=\frac{1}{16 \pi^{2}} \int b_{2}(A)|g|^{1 / 2} d^{4} x .
$$

Eq. (2.17) is the origin of the renormalisation group equation (1.2) we gave in the introduction. For Laplace type operators $A=-\nabla^{2}+E$, the expansion coefficient $b_{2}(A)$ is an invariant polynomial combination of the spacetime curvature and derivatives of $E$. In ref. [41], it was shown that the expansion coefficients remain polynomial for some classes of non-Laplacian operators relevant to the covariant effective action. In these cases, we can use $b_{2}(A)$ to read off the rescaling behaviour of the terms in the effective potential or the effective Lagrangian using the renormalisation group equation (1.2).

Since the scaling relations obtained from $b_{2}$ are local, and the difference between covariant and non-covariant effective actions vanishes when $\partial_{I} S=0$, we can conclude that

$$
\mu_{R} \frac{d \Gamma_{\mathrm{nc}}^{(1)}}{d \mu_{R}}-\mu_{R} \frac{d \Gamma_{E}^{(1)}}{d \mu_{R}}=f^{I} \partial_{I} S,
$$

where $f^{I}$ is a tensor polynomial expression of order $\hbar$ in the loop expansion. The noncovariant results are mostly known already, so this relation provides both a check on new covariant results and a possible route to finding the covariant $\beta$-functions. Furthermore, we can combine the terms at one loop order into

$$
\mu_{R} \frac{d \Gamma_{\mathrm{nc}}^{(1)}[\varphi]}{d \mu_{R}}=\mu_{R} \frac{d \Gamma_{E}^{(1)}\left[\varphi+f \ln \mu_{R}\right]}{d \mu_{R}}+O\left(\hbar^{2}\right) .
$$

Note that this does not imply that the approaches are equivalent, because the covariant action is covariant not invariant under field redefinitions. In the introduction, we described how to construct the renormalisation group corrected Lagrangian $\mathcal{L}^{\prime}$ from the rescaling behaviour of the action at one loop. Following the same procedure we see that the effective actions $\Gamma^{\prime}$ constructed from the covariant and the non-covariant effective actions are related by a field transformation,

$$
\Gamma_{\mathrm{nc}}^{\prime}[\varphi]=\Gamma_{E}^{\prime}[\varphi-f t],
$$

where $t=\ln (\phi / M)$. After expanding out again

$$
\Gamma_{\mathrm{nc}}^{\prime}[\varphi]=\Gamma_{E}^{\prime}[\varphi]-t f^{I} \partial_{I} \Gamma_{E}^{\prime}[\varphi]+O\left(t^{2} \hbar^{2}\right)
$$

At this point, we are unsure about the size of the $O\left(t^{2} \hbar^{2}\right)$ terms. In the one loop result (2.20), logarithms endanger the loop expansion when the logarithms are large. We corrected for this by using the effective couplings. The new remainder term depends on the combination $f t$. If we take the case where the remainder is small, and apply the corrected field equations $\partial_{I} \Gamma^{\prime}=0$, as we might use to calculate tunnelling amplitudes for example, then the non-covariant action has the same value as the covariant action. However, we see that for large field values the remainder term may be large, and there 
is a possible discrepancy between the non-covariant and covariant renormalisation group corrected potentials even when on shell.

To obtain the two representations of the covariant effective action given earlier, first split $\varphi^{I} \rightarrow\left(\xi^{I}, \theta^{I}\right)$ into non-gauge and pure-gauge directions. Decompose the operator $\mathcal{D}^{I} \mathcal{D}_{J} S$ as

$$
\mathcal{D}^{I} \mathcal{D}_{J} S=A=\left(\begin{array}{cc}
a & c \\
c^{\dagger} & d
\end{array}\right)
$$

Similarly decompose

$$
R^{I \alpha} R_{J \alpha}=B=\left(\begin{array}{ll}
0 & 0 \\
0 & b
\end{array}\right)
$$

Eq. (2.14) follows from this decomposition when we set $\lambda_{g}=1$ in the one-loop result (2.12). Noting that the non-zero eigenvalues of $R^{I \alpha} R_{J \alpha}$ and $R^{I \alpha} R_{I \beta}$ are identical,

$$
\Gamma^{(1)}=\frac{\hbar}{2 i} \operatorname{tr} \ln a+\frac{\hbar}{2 i} \operatorname{tr} \ln b-\frac{\hbar}{i} \operatorname{tr} \ln b=\frac{\hbar}{2 i} \operatorname{tr} \ln a-\frac{\hbar}{2 i} \operatorname{tr} \ln b .
$$

This recovers eq. (2.14).

For Landau gauge eq. (2.15), we start with the generalised zeta-function $\zeta(s, A)$. Consider

$$
\zeta\left(s, A+\lambda_{g} B\right)=\frac{1}{\Gamma(s)} \int_{0}^{\infty} d t t^{s-1} \operatorname{tr}\left(e^{-\left(A+\lambda_{g} B\right) t}\right)
$$

If we rescale $t$,

$$
\zeta\left(s, A+\lambda_{g} B\right)=\frac{\lambda_{g}^{-s}}{\Gamma(s)} \int_{0}^{\infty} d t t^{s-1} \operatorname{tr}\left(e^{-\left(B+\lambda_{g}^{-1} A\right) t}\right)
$$

Separate out the diagonal and non-diagonal parts,

$$
\operatorname{tr}\left(e^{-\left(B+\lambda_{g}^{-1} A\right) t}\right)=\operatorname{tr}\left(\exp \left[-\left(\begin{array}{cc}
\lambda_{g}^{-1} a & 0 \\
0 & b+\lambda_{g}^{-1} d
\end{array}\right) t\right] \exp \left[-\left(\begin{array}{cc}
0 & \lambda_{g}^{-1} c \\
\lambda_{g}^{-1} c^{\dagger} & 0
\end{array}\right) t\right]\right)
$$

Only the even powers of $\lambda_{g}^{-1}$ survive in the second exponential due to the trace. Of these, only the leading term survives in the large $\lambda_{g}$ limit, and after rescaling $t$ back,

$$
\zeta\left(s, A+\lambda_{g} B\right)=\zeta(s, a)+\lambda_{g}^{-s} \zeta\left(s, b+\lambda_{g}^{-1} d\right)+O\left(\lambda_{g}^{-s-2}\right)
$$

We use analytic continuation to $s=0$ and then the limit $\lambda_{g} \rightarrow \infty$,

$$
\begin{aligned}
\zeta\left(0, A+\lambda_{g} B\right) & \sim \zeta(0, a)+\zeta(0, b) \\
\zeta^{\prime}\left(0, A+\lambda_{g} B\right) & \sim \zeta^{\prime}(0, a)+\zeta^{\prime}(0, b)-\zeta(0, b) \ln \lambda_{g}
\end{aligned}
$$

So now the terms on the right hand side of (2.15) are

$$
\lim _{\lambda_{g} \rightarrow \infty} \frac{\hbar}{2 i} \operatorname{tr} \ln \left\{A+\lambda_{g} B\right\}-\frac{\hbar}{i} \operatorname{tr} \ln \left\{\lambda_{g}^{1 / 2} b\right\}=\frac{\hbar}{2 i} \operatorname{tr} \ln a-\frac{\hbar}{2 i} \operatorname{tr} \ln b
$$

Therefore the Landau gauge result eq. (2.15) is equal to eq. (2.25) which is equal to the gauge decomposition eq. (2.14). 


\section{$3 \quad$ The gravity-Higgs effective field theory}

We take the point of view that the gravity-Higgs sector is a low energy effective field theory for the spacetime metric $g_{\mu \nu}$ and the Higgs doublet field $\mathcal{H}$, in which non-renormalisable terms are assumed to be suppressed by inverse powers of the reduced Planck mass, $\kappa=$ $M_{p}^{-1}=(8 \pi G)^{1 / 2}[5]$. Since Higgs instability sets in at a scale below the Planck mass, the renormalisable couplings will be expected to play the most important role. During inflation, we suppose that the vacuum energy is dominated by an inflation field and takes some fixed value $V_{0}$, and then the expansion rate in the Higgs vacuum is determined by the Friedman equation $H^{2}=\kappa^{2} V_{0} / 3$.

For convenience, we replace the Higgs doublet by a set of four real scalars $\phi^{i}$, denoting the gauge invariant magnitude of the field by $\phi$ and the projection orthogonal to $\phi^{i}$ by $\delta_{i j}^{\perp}$. The Lagrangian density for the gravity-Higgs sector $\mathcal{L}_{g}$ with non-minimal coupling is

$$
\mathcal{L}_{g}(g, \phi)=\frac{1}{2 \kappa^{2}} U(\phi) R(g)|g|^{1 / 2}-\frac{1}{2} G_{i j}(\phi) g^{\mu \nu} \partial_{\mu} \phi^{i} \partial_{\nu} \phi^{j}|g|^{1 / 2}-V(\phi)|g|^{1 / 2},
$$

where $\partial_{\mu}$ denotes an ordinary spacetime derivative. The non-minimal coupling terms are contained in the function $U(\phi)$ multiplying the Ricci scalar $R$.

Each one of the scalar functions in the Lagrangian has an expansion in powers of $\kappa$,

$$
\begin{aligned}
V(\phi) & =V_{0}+\frac{1}{2} \mu^{2} \phi^{2}+\frac{1}{4} \lambda \phi^{4}+\frac{1}{6} \lambda_{6} \kappa^{2} \phi^{6}+\ldots \\
G_{i j}(\phi) & =\delta_{i j}+\alpha \kappa^{2} \delta_{i k} \delta_{j l} \phi^{k} \phi^{l}+\beta \kappa^{2} \delta_{i j}^{\perp} \phi^{2}+\ldots \\
U(\phi) & =1-\xi \kappa^{2} \phi^{2}+\ldots,
\end{aligned}
$$

Most of the results we obtain have been truncated to $O\left(\kappa^{2} \phi^{2}\right)$. We will use a wave function renormalisation to the keep the leading order behaviour in $R$ and $G_{i j}$ fixed. The anomalous dimensions will be denoted by $\gamma_{g}$ and $\gamma_{\phi}$ respectivly. This keeps the effective Planck scale fixed. Note that it is not possible to eliminate both coefficients $\alpha$ and $\beta$ by redefinitions of $\phi$ if $G_{i j}$ has a non-vanishing curvature tensor.

One of the questions we address is the effect of conformal rescaling of the metric from the original Jordan Frame to the Einstein frame to remove the $\xi$ term in the original Lagrangian. We set the metric to be $g_{E}=U(\phi) g$, then

$$
\mathcal{L}_{g}\left(g_{E}, \phi\right)=\frac{1}{2 \kappa^{2}} R\left(g_{E}\right)\left|g_{E}\right|^{1 / 2}-\frac{1}{2} G_{E i j}(\phi) g_{E}^{\mu \nu} \partial_{\mu} \phi^{i} \partial_{\nu} \phi^{j}\left|g_{E}\right|^{1 / 2}-V_{E}(\phi)\left|g_{E}\right|^{1 / 2},
$$

where

$$
\begin{aligned}
V_{E}(\phi) & =U^{-2} V(\phi) \\
G_{E i j}(\phi) & =U^{-1} G_{i j}+\frac{3}{2} \kappa^{-2} U^{-2} \frac{\partial U}{\partial \phi^{i}} \frac{\partial U}{\partial \phi^{j}}
\end{aligned}
$$

In a covariant theory it should be possible to calculate the beta functions by transforming to the Einstein frame, rescaling the effective action, and transforming back to the Jordan frame. 
If we expand the Einstein frame theory in powers of $\kappa$ we have relationships between the sets of Einstein frame and Jordan frame parameters,

$$
\begin{aligned}
\mu_{E}^{2} & =\mu^{2}+4 \xi^{\prime} \kappa^{2} V_{0} \\
\lambda_{E} & =\lambda+4 \xi^{\prime} \kappa^{2} \mu^{2} \\
\xi_{E} & =\xi-\xi^{\prime} .
\end{aligned}
$$

Note that we have used a different $\xi^{\prime}$ for the conformal transformation. Since we have adopted a covariant quantisation approach, these relations also hold for the running couplings up to field renormalisation factors. We differentiate the relations with respect to the renormalisation scale keeping $\xi^{\prime}$ fixed, and then set $\xi^{\prime}=\xi$ at the end,

$$
\begin{aligned}
\tilde{\beta}_{\mu^{2}}\left(0, \lambda_{E}, \mu_{E}^{2}, \ldots\right) & =\tilde{\beta}_{\mu^{2}}\left(\xi, \lambda, \mu^{2}, \ldots\right)+4 \xi \kappa^{2} \tilde{\beta}_{V_{0}}\left(\xi, \lambda, \mu^{2}, \ldots\right) \\
\tilde{\beta}_{\lambda}\left(0, \lambda_{E}, \mu_{E}^{2}, \ldots\right) & =\tilde{\beta}_{\lambda}\left(\xi, \lambda, \mu^{2}, \ldots\right)+4 \xi \kappa^{2} \tilde{\beta}_{\mu^{2}}\left(\xi, \lambda, \mu^{2}, \ldots\right) \\
\tilde{\beta}_{\xi}\left(0, \lambda_{E}, \mu_{E}^{2}, \ldots\right) & =\tilde{\beta}_{\xi}\left(\xi, \lambda, \mu^{2}, \ldots\right)
\end{aligned}
$$

The beta functions $\tilde{\beta}$ include anomalous dimension factors, for example

$$
\tilde{\beta}_{\xi}=\beta_{\xi}-2 \gamma_{\phi} \xi-\gamma_{g} \xi .
$$

These relations can be used to evaluate covariant beta functions for non-zero curvature coupling if we have results for minimal coupling.

Already, an unexpected result follows from (3.13), namely that the one-loop $\beta_{\xi}$ for gravity-Higgs theory is independent of $\xi$ at order $\kappa^{0}$. Paradoxically, the quantum theory of scalar fields on a curved background gives $\beta_{\xi} \propto 6 \xi-1$ [42]. The $\xi$ dependence must cancel when we include quantum gravity and require field-covariance of the effective action. Subsequent results will confirm this using explicit calculations.

\section{Expansions of the gravity-Higgs action}

We will give results for the second order variations of the gravity-Higgs effective theory which are needed to evaluate the beta functions. Most of the details have been left out because these are already covered in the literature, particularly in the work of Barvinsky et al. [43-45]). We use the Jordan frame formulation and then the covariant formulation can be checked by verifying the relations between the beta functions give in eqs. (3.11)-(3.13).

Variations in field space can be combined into metric and scalar directions, and we rescale these to have the same dimensions, i.e.

$$
\partial_{I} S=\left(2 \kappa \frac{\delta S}{\delta g_{\mu \nu}}, \frac{\delta S}{\delta \phi^{i}}\right)
$$

The second-order variation of the action gives a second order differential operator,

$$
-\mathcal{D}_{I} \mathcal{D}_{J} S=-\partial_{I} \partial_{J} S+\Gamma^{K}{ }_{I J} \partial_{K} S=-\mathcal{G}_{I J} \nabla^{2}-\mathcal{P}^{\alpha \beta}{ }_{I J} \nabla_{\alpha} \nabla_{\beta}+E_{I J},
$$

There are three important tensors in this expression which will be presented below: $\mathcal{G}_{I J}$ is the metric on field space, $\mathcal{P}_{I J}^{\alpha \beta}$ projects out the gauge-fixed directions, and $E_{I J}$ is an 
effective mass term. The metric is used to construct the Levy-Civita connection by the usual expression,

$$
\Gamma^{I}{ }_{J K}=\frac{1}{2} \mathcal{G}^{I L}\left(\partial_{K} \mathcal{G}_{L J}+\partial_{J} \mathcal{G}_{L K}-\partial_{L} \mathcal{G}_{J K}\right)
$$

When writing down local operators like $\mathcal{G}_{I J}$ we usually omit delta function terms.

\subsection{First order variations}

The first order variation of the action defines the background field equations for the gravitational and Higgs fields, which will be denoted by $F^{\mu \nu}$ and $F_{i}$,

$$
\begin{gathered}
F^{\mu \nu}=-\frac{\kappa}{\sqrt{g}} \frac{\delta S}{\delta g_{\mu \nu}}=U G^{\mu \nu}-U^{; \mu \nu}+g^{\mu \nu} U_{\rho}^{; \rho}-\kappa^{2} T^{\mu \nu}, \\
F_{i}=-\frac{1}{\sqrt{g}} \frac{\delta S}{\delta \phi^{i}}=-\frac{1}{2 \kappa^{2}} R U_{, i}-D_{\mu}\left(G_{i j} \nabla^{\mu} \phi^{i}\right)+V_{, i},
\end{gathered}
$$

where $D_{\mu}$ is a covariant derivative for the metric $G_{i j}$,

$$
D_{\mu} \delta \phi^{i}=\partial_{\mu} \delta \phi^{i}-\Gamma_{j k}^{i}\left(\partial_{\mu} \phi^{j}\right) \delta \phi^{k}
$$

Note that $F^{\mu \nu}$ has been scaled so that $F^{\mu \nu}=0$ resembles the usual Einstein equation.

\subsection{Second order variations}

For simplicity, the background scalar field will be assumed constant. The second order variation $-\partial_{I} \partial_{J} S_{g}$ has derivative terms

$$
\left(\begin{array}{cc}
-U g^{(\mu \nu)(\rho \sigma)} \nabla^{2}+U P^{\alpha \beta(\mu \nu)(\rho \sigma)} \nabla_{\alpha} \nabla_{\beta}-\kappa^{-1} U_{, j}\left(\nabla^{\mu} \nabla^{\nu}-g^{\mu \nu} \nabla^{2}\right) \\
-\kappa^{-1} U_{, i}\left(\nabla^{\rho} \nabla^{\sigma}-g^{\rho \sigma} \nabla^{2}\right) & -G_{i j} \nabla^{2}
\end{array}\right)|g|^{1 / 2},
$$

and a potential term,

$$
E_{g I J}=\left(\begin{array}{cc}
E_{g}^{(\mu \nu)(\rho \sigma)} & \kappa g^{\mu \nu} V_{, j} \\
\kappa g^{\rho \sigma} V_{, i} & V_{, i j}-\frac{1}{2} R \kappa^{-2} U_{, i j}
\end{array}\right)|g|^{1 / 2},
$$

Two important tensors in the kinetic terms are the DeWitt metric,

$$
g^{(\mu \nu)(\rho \sigma)}=\frac{1}{2}\left(g^{\mu \rho} g^{\nu \sigma}+g^{\mu \sigma} g^{\nu \rho}-g^{\mu \nu} g^{\rho \sigma}\right),
$$

and another tensor which will also appear in the gauge-fixing terms below,

$$
P^{\alpha \beta(\mu \nu)(\rho \sigma)}=2 g_{\gamma \delta} g^{(\alpha \gamma)(\mu \nu)} g^{(\beta \delta)(\rho \sigma)} .
$$

The mass-like gravity terms are

$$
E_{g}^{(\mu \nu)(\rho \sigma)}=-2 U R^{\dot{\mu} \rho \dot{\nu} \sigma}+2 U R^{\dot{\mu} \rho} g^{\dot{\nu} \sigma}+U R_{T}^{\mu \nu} g^{\rho \sigma}+U g^{\mu \nu} R_{T}^{\rho \sigma}-4 U R_{T}^{\dot{\mu} \rho} g^{\dot{\nu} \sigma}-2 \kappa^{2} V g^{(\mu \nu)(\rho \sigma)} .
$$

Dots over indices indicate symmetrisation in those indices and a subscript $T$ denotes the trace-free part of the tensor. The terms have been organised this way to isolate terms 
which vanish when the differential operator is applied to transverse traceless perturbations and terms which remain.

Gauge-fixing terms have to be included in the action, and following Barvinski [43] we take

$$
\mathcal{L}_{\text {gf }}=-\lambda_{g} U g_{\mu \nu} \chi^{\mu} \chi^{\nu}
$$

where

$$
\chi^{\mu}=\frac{1}{2 \kappa}\left(g^{(\mu \nu)(\rho \sigma)} \nabla_{\nu} \delta g_{\rho \sigma}-U^{-1} U_{, i} \nabla^{\mu} \delta \phi^{i}\right)
$$

The gauge-fixing term gives a contribution to the second-order variation of,

$$
-\partial_{I} \partial_{J} S_{g f}=-\lambda_{g}\left(\begin{array}{cc}
U P^{\alpha \beta(\mu \nu)(\rho \sigma)} \nabla_{\alpha} \nabla_{\beta} & -\kappa^{-1} U_{, j} g^{(\mu \nu)(\alpha \beta)} \nabla_{\alpha} \nabla_{\beta} \\
-\kappa^{-1} U_{, i} g^{(\rho \sigma)(\alpha \beta)} \nabla_{\alpha} \nabla_{\beta} & \frac{1}{2} \kappa^{-2} U^{-1} U_{, i} U_{, j} \nabla^{2}
\end{array}\right)|g|^{1 / 2},
$$

We have enough information now to obtain the field-space metric $\mathcal{G}_{I J}$. We will do this by requiring the operator to have Laplacian form in the gauge-fixed directions, i.e.

$$
-\left(\partial_{I} \partial_{J} S_{g}\right) \delta \varphi^{I}=-\mathcal{G}_{I J} \nabla^{2} \delta \varphi^{J}+E_{I J} \delta \varphi^{J}
$$

when $\chi^{\mu}(\delta \varphi)=0$. Note that variations of the gauge-fixing term vanish when applied to the gauge-fixed directions, and so an arbitrary amount of $\partial_{I} \partial_{J} S_{g f}$ can be added to the differential operator. However, $\left(\partial_{I} \partial_{J} S_{g}\right)+\lambda_{g}^{-1}\left(\partial_{I} \partial_{J} S_{g f}\right)$ is the unique combination of the second order variations that has Laplacian form. The coefficient of $-\nabla^{2}$ in this combination is therefore the field-space metric, and this gives

$$
\mathcal{G}_{I J}=\left(\begin{array}{cc}
U g^{(\mu \nu)(\rho \sigma)} & -\frac{1}{2} \kappa^{-1} U_{, j} g^{\mu \nu} \\
-\frac{1}{2} \kappa^{-1} U_{, i} g^{\rho \sigma} & G_{i j}+\frac{1}{2} \kappa^{-2} U^{-1} U_{, i} U_{, j}
\end{array}\right)|g|^{1 / 2} .
$$

(We also obtain exactly the same result using $\partial_{I} \chi^{\alpha}=\lambda_{g}^{1 / 2} R_{I}{ }^{\alpha}$ for the $R_{\xi}$ gauges as in section 2. This is a special feature of the gauge fixing term (4.13), and for other choices it becomes necessary to combine information from both gauge-fixed and pure gauge directions to obtain the metric). For future reference, the inverse metric is given by

$$
\mathcal{G}^{I J}=\left(\begin{array}{cc}
U^{-1} g_{(\mu \nu)(\rho \sigma)}+\frac{1}{4} \kappa^{-2} U^{-1} U_{, k} U^{, k} W^{-1} g_{\mu \nu} g_{\rho \sigma} & -\frac{1}{2} \kappa^{-1} W^{-1} U^{, j} g_{\mu \nu} \\
-\frac{1}{2} \kappa^{-1} W^{-1} U^{, i} g_{\rho \sigma} & G^{i j}-\frac{3}{2} W^{-1} \kappa^{-2} U^{, i} U^{, j}
\end{array}\right)|g|^{-1 / 2}
$$

where $W=U+\frac{3}{2} \kappa^{-2} U^{-1} U_{, i} U^{, i}$.

Finally, comparing to the expression (4.2), we can also read off the tensor $\mathcal{P}^{\alpha \beta}{ }_{I J}$,

$$
\mathcal{P}^{\alpha \beta}{ }_{I J}=\left(\begin{array}{cc}
U P^{\alpha \beta(\mu \nu)(\rho \sigma)} & -\kappa^{-1} U_{, j} g^{(\mu \nu)(\alpha \beta)} \\
-\kappa^{-1} U_{, i} g^{(\rho \sigma)(\alpha \beta)} & \frac{1}{2} \kappa^{-2} U^{-1} U_{, i} U_{, j}
\end{array}\right)|g|^{1 / 2} .
$$

The variations are considerably simpler in the Einstein frame $U \equiv 1$. Indeed, one of the motivations for considering covariant approaches is to ensure that the Einstein frame result can always be used reliably. However, just this once, we are going to verify that the covariant result is independent of the choice of conformal frame. 


\subsection{Vilkovisky-DeWitt corrections}

The Levy-Civita connection is given by the usual expression (4.3). The connection converts the scalar derivatives $V_{, i j}$ into covariant derivatives $V_{; i j}$, and adds extra terms to the differential operator (4.2). For simplicity, we just quote the contributions up to $O\left(\kappa^{2}\right)$, and take the spacetime curvature to be of order $\kappa^{2}$ and the scalar derivatives $V_{, i}$ of order $\kappa$, then

$$
\Gamma^{K}{ }_{I J} \partial_{K} S_{g}=\left(\begin{array}{cc}
E_{\Gamma}(\mu \nu)(\mu \nu) & -\frac{1}{4} \kappa\left(2 V_{, j}-\kappa^{-2} R U_{, j}\right) g^{\mu \nu} \\
-\frac{1}{4} \kappa\left(2 V_{, i}-\kappa^{-2} R U_{, i}\right) g^{\rho \sigma} & \frac{1}{2}\left(G_{i j}+\kappa^{-2} U_{; i j}\right)\left(R-4 \kappa^{2} V\right)
\end{array}\right),
$$

where

$$
E_{\Gamma}^{(\mu \nu)(\rho \sigma)}=2 U g^{\dot{\mu} \rho} R_{T}^{\dot{\nu} \sigma}-\frac{1}{2} U R_{T}^{\mu \nu} g^{\rho \sigma}-\frac{1}{2} U g^{\rho \sigma} R_{T}^{\mu \nu} .
$$

Eq. (4.19) is exact when in the minimally coupled case $U=1$. In the non-minimally coupled case, when the Levy-Civita connection term is combined with the mass terms from the second variation of the action (4.8), we see that the curvature coupling terms $U_{; i j} R$ cancel. In particular, the $2 \xi \kappa^{2} R$ term which would contribute $\xi$ dependence to the beta-function $\beta_{\xi}$ has been cancelled off by the Vilkovisky-DeWitt corrections.

\section{Gravity-Higgs mode expansions}

We argued in section 2 that the scaling behaviour of the gravity-scalar effective action can be expressed in terms of spacetime invariant tensor combinations. General expressions for these combinations are known from heat kernel methods for a wide range of second order operators $[30,41]$, but there are some non-Laplace type operators where the general results are not yet available. Furthermore, it can be very cumbersome applying these general results. A more practical approach is to use a direct evaluation of the the generalised zeta function on a simple manifold for a simple operator, for example gravity with a single scalar field on the sphere $[46,47]$, and read off the relevant coefficients.

On the sphere $S_{4}$, the curvature is given in terms of the Ricci scalar

$$
R_{\mu \nu \rho \sigma}=\frac{1}{12} R\left(g_{\mu \rho} g_{\nu \sigma}-g_{\mu \sigma} g_{\nu \rho}\right),
$$

and the radius of the sphere is $\sqrt{12 / R}$. Consider a single constant scalar field $\phi$ with Euclidean Lagrangian

$$
\mathcal{L}_{E}=\frac{1}{2} K(\phi)(\nabla \phi)^{2}+V(\phi)-\frac{1}{\kappa^{2}} U(\phi) R
$$

The second order operator for the Euclidean theory is

$$
\mathcal{D}^{I} \mathcal{D}_{J} S_{E}=-\delta^{I}{ }_{J} \nabla^{2}+\left(\lambda_{g}-1\right) \mathcal{R}^{I \alpha} \mathcal{R}_{J \alpha}+E^{I}{ }_{J},
$$

where the general expressions for $\mathcal{R}^{I \alpha} \mathcal{R}_{J \alpha}$ and $E^{I}{ }_{J}$ where given in section 4 . For a single field, we replace $V_{, i}$ by $V^{\prime}$ and $V_{; i j}$ by the covariant derivative with metric $K(\phi)$,

$$
V^{\prime \prime}=K^{1 / 2}\left(K^{-1 / 2} V^{\prime}\right)^{\prime}
$$


The differential operators can be diagonalised by expanding the fields in a basis of $S_{4}$ orthonormal harmonics: scalar modes $h^{S}$, transverse vector modes $h_{\mu}^{V}$ and transversetraceless tensor modes $h^{T}{ }_{\mu \nu}$. Transverse modes are divergence free, i.e. $\nabla^{\mu} h^{V}{ }_{\mu}=0$. The eigenvalues of $-\nabla^{2}$ for the respective modes are $\lambda_{S}, \lambda_{V}$ and $\lambda_{T}$. Modes can be traded up into higher rank tensors by applying derivatives to the basic set of harmonics. The general decomposition of the metric plus scalar field into the basis of harmonic functions and their derivatives is given by mode sums with coefficients $x^{I}$,

$$
\delta g_{\mu \nu}=2 \kappa \sum_{\text {modes }}\left\{x^{1} h_{\mu \nu}^{T}+2 x^{2} \nabla_{(\mu} h_{\nu)}^{V}+x^{3} \nabla_{\mu \nu} h^{S}+x^{4} g_{\mu \nu} h^{S}\right\}, \quad \delta \phi^{i}=\sum_{\text {modes }} x^{5} h^{S},
$$

where $\nabla_{\mu \nu}=\nabla_{\mu} \nabla_{\nu}-\frac{1}{4} g_{\mu \nu} \nabla^{2}$. In the ghost sector, there is a similar decomposition,

$$
c_{\mu}=\sum_{\text {modes }}\left\{y^{1} h_{\mu}^{V}+y^{2} \nabla_{\mu} h^{S}\right\} .
$$

The eigenvalues of the derived modes change due to non-commutation of the covariant derivatives, for example

$$
-\nabla^{2}\left(\nabla_{(\mu} h_{\nu)}^{V}\right)=\left(\lambda_{V}-\frac{5}{12} R\right) \nabla_{(\mu} h_{\nu}^{V}
$$

The derived modes are not normalised, but their normalisation can be deduced from the original harmonic, for example

$$
4 \int \nabla_{(\mu} h_{\nu)}^{V} \nabla^{(\mu} h^{V \nu)}|g|^{1 / 2} d^{4} x=2\left(\lambda_{V}-\frac{1}{4} R\right) .
$$

The action of the operators and products in the harmonic basis for a given set of eigenvalues can be represented now by $5 \times 5$ matrices, which are given in appendix $\mathrm{A}$.

At this point, we would like to stress an important point that the matrices $\mathcal{D}_{I} \mathcal{D}_{J} S_{E}$ are not positive definite, so there are directions which decrease the Euclidean action and invalidate the path integral approach. This is the famous conformal mode problem of Euclidean quantum gravity. However, the matrices representing $\Pi \mathcal{D}_{I} \mathcal{D}_{J} S_{E} \Pi$, and $\mathcal{D}_{I} \mathcal{D}_{J} S_{E}+\lambda_{g} R_{I}^{\alpha} R_{J \alpha}$ for sufficiently large $\lambda_{g}$, are both positive definite and the path integral can be defined. This is the solution to the conformal mode problem of Euclidean quantum gravity referred to in ref. [41].

The renormalisation scale dependence of the one-loop effective action can be calculated in two different ways, and the comparison gives a check on the accuracy of the result. The first way is by gauge decomposition,

$$
\mu_{R} \frac{d \Gamma_{E}^{(1)}}{d \mu_{R}}=-\zeta\left(0, \Pi\left(\mathcal{D}^{I} \mathcal{D}_{J} S\right) \Pi\right)+\zeta\left(0, \mathcal{R}^{I \alpha} \mathcal{R}_{I \beta}\right)
$$

The second version is Landau gauge,

$$
\mu_{R} \frac{d \Gamma_{E}^{(1)}}{d \mu_{R}}=\lim _{\lambda_{g} \rightarrow \infty}\left\{-\zeta\left(0, \mathcal{D}^{I} \mathcal{D}_{J} S+\lambda_{g} R^{I \alpha} R_{J \alpha}\right)+2 \zeta\left(0, \lambda_{g}^{1 / 2} \mathcal{R}^{I \alpha} \mathcal{R}_{I \beta}\right)\right\}
$$


In each case, the eigenvalues are evaluated by diagonalising the matrices and the generalised zeta functions are defined for $s>2$ by series,

$$
\zeta(s, A)=\sum_{\lambda} \lambda^{-s}
$$

Spherical harmonic eigenvalues are all quadratic polynomials in a single 'angular momentum' index $n$. After diagonalisation, the eigenvalues are algebraic functions of the spherical harmonic eigenvalues, but standard zeta-function methods can be modified to analytically continue and evaluate $\zeta(0, A)[48]$.

For example, in the transverse-traceless tensor sector $I=J=1$, the eigenvalues are the same for either method,

$$
\lambda=\lambda_{T}+U^{-1} m_{T}^{2}
$$

The tensor eigenvalues are given in appendix B, and after analytic continuation using (B.16),

$$
\zeta\left(0,\left(\mathcal{D}^{2} S\right)^{1}{ }_{1}\right)=-\frac{1}{18}+20 \frac{m_{T}^{2}}{U R}+60 \frac{m_{T}^{4}}{U^{2} R^{2}}
$$

Contributions to the beta-functions from the transverse-traceless tensors can be obtained from

$$
\mu_{R} \frac{d \mathcal{L}_{E}^{(1)}}{d \mu_{R}}=-\frac{b_{2}}{16 \pi^{2}},
$$

where the adiabatic expansion coefficient $b_{2}$ can be extracted from

$$
b_{2}=\frac{16 \pi^{2}}{\text { Volume } S_{4}} \zeta\left(0,\left(\mathcal{D}^{2} S\right)^{1}{ }_{1}\right)=\frac{R^{2}}{24} \zeta\left(0,\left(\mathcal{D}^{2} S\right)^{1}{ }_{1}\right)
$$

After substituting for $m_{T}^{2}$ (see appendix B), the tensor mode contribution to $b_{2}$ becomes

$$
b_{2}=\frac{719}{432} R^{2}-\frac{25}{3} \frac{\kappa^{2} R V}{U}+10 \frac{\kappa^{4} V^{2}}{U^{2}} .
$$

For example, with $U=1-\xi \kappa^{2} \phi^{2}$, we have a contribution to $\beta_{\xi}$ from expanding the second term in powers of $\kappa$,

$$
\beta_{\xi}-2 \gamma_{\phi} \xi-\gamma_{g} \xi=2 \operatorname{coeff}\left(b_{2}, R \phi^{2}\right)=\frac{50}{3} \kappa^{4} V_{0}+O\left(\kappa^{6}\right) .
$$

Other contributions to the beta functions can be obtained in a similar way from the matrices given above, but the details are lengthy and unilluminating. The results given below have been obtained using MAPLE and most of them have been checked by hand. We will give results for the contributions to the beta functions from the Higgs background direction and the gravitational sector with which it mixes. Other contributions from the Higgs components perpendicular to the background direction ('Goldstone-like modes') will be given in a later section.

Tables 1 and 2 show results at leading order for small $\kappa^{4} V_{0}$, assuming that the curvature $R$ and the mass square $\mu^{2}$ are of order $\kappa^{2} V_{0}$. These choices are consistent when the application is to Higgs stability, where the curvature of the universe $R \approx 4 \kappa^{2} V_{0}$ and the 


\begin{tabular}{|lll|}
\hline & Jordan frame & Einstein frame \\
\hline $16 \pi^{2} \beta_{\xi}$ & $(6 \xi-1) \lambda$ & $-\lambda$ \\
$16 \pi^{2} \beta_{\mu^{2}}$ & $6 \mu^{2} \lambda$ & $6\left(\mu^{2}+4 \xi \kappa^{2} V_{0}\right) \lambda$ \\
$16 \pi^{2}\left(\beta_{\mu^{2}}+4 \kappa^{2} V_{0} \beta_{\xi}\right)$ & $6\left(\mu^{2}+4 \xi \kappa^{2} V_{0}\right) \lambda-4 \lambda \kappa^{2} V_{0}$ & $6\left(\mu^{2}+4 \xi \kappa^{2} V_{0}\right) \lambda-4 \lambda \kappa^{2} V_{0}$ \\
\hline
\end{tabular}

\begin{tabular}{|ll|}
\hline & Covariant \\
\hline $16 \pi^{2} \beta_{\xi}$ & $2 \lambda$ \\
$16 \pi^{2} \beta_{\mu^{2}}$ & $6\left(\mu^{2}+4 \xi \kappa^{2} V_{0}\right) \lambda-12 \lambda \kappa^{2} V_{0}$ \\
$16 \pi^{2}\left(\beta_{\mu^{2}}+4 \kappa^{2} V_{0} \beta_{\xi}\right)$ & $6\left(\mu^{2}+4 \xi \kappa^{2} V_{0}\right) \lambda-4 \lambda \kappa^{2} V_{0}$ \\
\hline
\end{tabular}

Table 1. $\beta$-functions for the curvature coupling and the mass of a gravity coupled scalar field at leading order for small $\kappa^{4} V_{0}$. The Jordan frame result has been calculated directly from the original action. The Einstein frame result is obtained by transforming the action to the Einstein frame. The covariant result uses a geodesic expansion on field space and is independent of the frame used. The renormalisation group flow of $\mu^{2}+4 \kappa^{2} V_{0} \xi$ is the same for each of these approaches.

\begin{tabular}{|lll|}
\hline & Jordan frame & Covariant \\
\hline $16 \pi^{2} \beta_{\lambda}$ & $18 \lambda^{2}$ & $18 \lambda^{2}$ \\
$16 \pi^{2} \beta_{6}$ & $90 \lambda \lambda_{6}-18 \lambda^{2}\left(2-8 \xi+18 \xi^{2}\right)$ & $90 \lambda \lambda_{6}-18 \lambda^{2}\left(1-7 \xi+24 \xi^{2}\right)$ \\
$16 \pi^{2} \gamma_{g}$ & $-\frac{1}{3} \kappa^{2} \mu^{2}+2 \kappa^{2}\left(\mu^{2} \xi-4 \kappa^{2} V_{0}\right)$ & $\frac{2}{3}\left(\mu^{2}+4 \xi \kappa^{2} V_{0}\right) \kappa^{2}-\frac{52}{3} \kappa^{4} V_{0}$ \\
\hline
\end{tabular}

Table 2. $\beta$-functions for the quartic scalar self-coupling $\lambda$ and the sixth order scalar self-coupling $\lambda_{6}$ of a gravity coupled scalar field at leading order for small $\kappa^{4} V_{0}$. The wave function renormalisation of the metric $\gamma_{g}$ is given at order $\kappa^{4} V_{0}$. The Jordan frame results have been calculated directly from the original action. The Einstein frame results are obtained by transforming the action to the Einstein frame. The covariant result uses a geodesic expansion on field space and is independent of the frame used.

Higgs mass is negligible. Contributions to the beta functions from the gravitational perturbations, like the transverse traceless tensor modes discussed above, enter only at order $\kappa^{4} V_{0}$, in agreement with the conclusion of ref. [22]. However, quantum gravity does has an effect at leading order through the Vilkovisky-DeWitt connection terms in the operators.

The first thing to notice in table 1 is the absence of $\xi$ terms for $\beta_{\xi}$ in the Einstein frame and the covariant results. The reason for this in the Einstein frame is obvious, since the $\xi R \phi^{2}$ term has been eliminated by the conformal transformation, and $\xi$ appears in the Einstein frame scalar potential $V_{J} / U^{2}$ instead. The absence of $\xi$ in the covariant results follows from the beta-function relations (3.11)-(3.13). In the explicit calculation, the leading order contribution to $\beta_{\xi}$ from $R U^{\prime \prime}$ in the mass matrix (A.3) cancels with the Vilkovisky-DeWitt correction.

In table 1 we see that the renormalisation group flow of $\mu^{2}+4 \kappa^{2} V_{0} \xi$ is the same in all the different approaches. For Higgs stability, $R \approx 4 \kappa^{2} V_{0}$, and the effective square mass of the Higgs field $\mu^{2}+\xi R \approx \mu^{2}+4 \kappa^{2} V_{0} \xi$. This is the crucial combination which determines whether the Higgs can survive in the false vacuum during early universe inflation, as we 
shall see later in section 7. The non-covariant formulation in the Jordan or the Einstein frame therefore gives the same outcome for stability as the covariant approach, at least for small values of $\kappa^{4} V_{0}$.

\section{Gauge bosons, Goldstone modes and fermions}

We turn now to gauge bosons, Goldstone modes and fermion contributions to the effective action for the scalar field on a curved spacetime background. In this case, there are no background gauge fields and the gauge modes decouple from the graviton and scalar modes of the previous section. Quantum gravity still has an effect via the Vilkovisky-DeWitt connection term. We will describe the calculation in the Einstein frame, using the betafunction relations (3.11)-(3.13) to extend the results to the Jordan frame. All the results have been checked using a direct Jordan frame calculation.

The gauge-Goldstone mode action which we use is

$$
\mathcal{L}_{g}=-\frac{1}{4} F_{a \mu \nu} F^{a \mu \nu}|g|^{1 / 2}-\frac{1}{2} \delta_{i j}^{\perp}\left(D_{\mu} \phi\right)^{i}\left(D^{\mu} \phi\right)^{j}|g|^{1 / 2}-V(\phi)|g|^{1 / 2},
$$

where $D_{\mu} \phi=\nabla_{\mu} \phi-g A_{a \mu} T^{a} \phi$ and $\delta_{i j}^{\perp}$ is orthogonal to the background Higgs direction used in the previous section.

For the Electroweak theory in particular, variations in the $W, Z$ and the photon directions decouple. In one of the two ' $W$ ' directions for example, $\varphi^{I}=\left(A_{W \mu}, \phi^{W}\right)$ and the second variation of the action with respect to the field variables is

$$
-\partial_{I} \partial_{J} S_{g}=\left(\begin{array}{cc}
-g^{\mu \nu} \nabla^{2}+\nabla^{\mu} \nabla^{\nu}+R^{\mu \nu}+m_{W}^{2} & -m_{W} \nabla^{\mu} \\
m_{W} \nabla^{\nu} & -\nabla^{2}+\mu^{2}+\lambda \phi^{2}
\end{array}\right)|g|^{1 / 2},
$$

where $m_{W}=g \phi / 2$. The second variation in the $Z$ direction is similar, with $m_{Z}=\left(g^{2}+\right.$ $\left.g^{\prime 2}\right)^{1 / 2} \phi / 2$, where $g^{\prime}$ is the coupling to the U(1) gauge field. The name 'Goldstone modes' has been used, although the modes are in fact massive because the background scalar field is not at the minimum of the potential.

We can read off the local field-space metric from the coefficients of the Laplacian terms, $\mathcal{G}_{I J}=\operatorname{diag}\left(g^{\mu \nu}, 1\right)|g|^{1 / 2}$. The $R_{\xi}$ gauges from section 2 correspond to the gauge-fixing functional

$$
\chi^{\alpha}=\nabla^{\mu} A_{a \mu}-g \phi^{T} T_{a} \delta \phi .
$$

The gauge-fixing contribution to the action is then

$$
R_{I \alpha} R_{J}^{\alpha}=\left(\begin{array}{cc}
\delta^{a b} \nabla^{\mu} \nabla^{\nu} & m_{W} \nabla^{\mu} \\
-m_{W} \nabla^{\nu} & m_{W}^{2}
\end{array}\right)|g|^{1 / 2} .
$$

The ghost operator in the gauge direction associated with the $W$ is

$$
Q^{\alpha}{ }_{\beta}=-\nabla^{2}+m_{W}^{2}
$$

There are two ghosts associated with the $W$ direction and one with the $Z$ direction. 
In the covariant approach, there are connection terms in the differential operator $\mathcal{D}_{I} \mathcal{D}_{J} S$ because the field-space metric $\mathcal{G}_{I J}$ depends on the spacetime metric, leading to a connection coefficient $\Gamma^{K}{ }_{I J}$ with $K$ in the metric direction. The contribution to the operator is $E_{\Gamma I J}=\Gamma_{I J}^{K} \partial_{K} S$,

$$
E_{\Gamma I J}=\left(\begin{array}{cc}
-G_{\mu \nu}-\kappa^{2} V g_{\mu \nu} & 0 \\
0 & \frac{1}{2}\left(R-4 \kappa^{2} V\right)
\end{array}\right)|g|^{1 / 2},
$$

where $G_{\mu \nu}+\kappa^{2} V g_{\mu \nu}=0$ is the Einstein equation when $\phi$ is constant. Note that the gauge and the scalar components of the operator all have a mass term containing $m_{W}^{2}+R / 2$ when the different contributions are added together.

The scaling behaviour of the one-loop action can be found as before by taking the spacetime background to be the Euclidean four-sphere. An important new consideration for the gauge boson beta-functions is the Higgs field wave function renormalisation at one loop,

$$
16 \pi^{2} \gamma_{\phi}=-\frac{3}{4} g_{\mathrm{tot}}^{2}+O\left(\kappa^{4} V_{0}\right)
$$

where $g_{\text {tot }}^{2}=3 g^{2}+g^{\prime 2}$. The leading term is simply the flat space result in Landau gauge. The absence of contributions at order $\kappa^{2}$ can be seen from relation (2.19), which expresses the difference between covariant and non-covariant results in terms of a tensor polynomial $f$ depending on the operator and the background field Einstein equation (4.4),

$$
\mu_{R} \frac{\partial \mathcal{L}}{\partial \mu_{R}}-\mu_{R} \frac{\partial \mathcal{L}_{n c}}{\partial \mu_{R}}=f \times\left[\kappa^{2}(\nabla \phi)^{2}+4 \kappa^{2} V-R\right]
$$

The only constant in the operator (6.2) which could contribute to $f$ is $\mu^{2}$. This would give a $\kappa^{2} \mu^{2}(\nabla \phi)^{2}$ term, but this is of order $\kappa^{4} V_{0}$ given our assumptions about $\mu^{2}$.

Results are given in tables 3 and 4 . The covariant beta-functions are independent of frame, and differ from the non-covariant expressions. As before, the two approaches agree on the combination which is important for Higgs stability, $\mu^{2}+4 \kappa^{2} V_{0} \xi$. There are differences in the sixth order coupling $\lambda_{6}$, but this only enters the Higgs field equations at $O\left(\kappa^{4} V_{0}\right)$.

We finish of with the top quark as an example of a fermion field. It is far from clear how the covariant approach generalises to fermion fields, so we take the minimalist approach and leave off any extra contributions to the effective action. The results are then checked for consistency against the covariant beta function relations (3.11)-(3.13). The beta functions are old results, but we repeat them here for completeness. The rescaling behaviour of the effective action due to the quark field is

$$
\mu_{R} \frac{d \Gamma_{E}^{(1)}}{d \mu_{R}}=3 \zeta\left(0,-\nabla^{2}+m_{t}^{2}+\frac{1}{4} R\right)
$$

where $m_{t}=y \phi / \sqrt{2}$ is the top quark mass and $\nabla_{\mu}$ is the covariant derivative acting on Dirac fields. Fermion fields take the positive sign and the pre-factor takes into account 


\begin{tabular}{|ll|}
\hline & Jordan frame \\
\hline $16 \pi^{2} \beta_{\xi}$ & $-\frac{1}{4}(6 \xi-1) g_{\text {tot }}^{2}+(6 \xi-1) \lambda$ \\
$16 \pi^{2} \beta_{\mu^{2}}$ & $-\frac{1}{2} \mu^{2} g_{\text {tot }}^{2}+6 \lambda \mu^{2}$ \\
$16 \pi^{2}\left(\beta_{\mu^{2}}+4 \kappa^{2} V_{0} \beta_{\xi}\right)$ & {$\left[6 \mu_{E}^{2}-4 \kappa^{2} V_{0}\right] \lambda-\frac{1}{2}\left[\mu_{E}^{2}-2 \kappa^{2} V_{0}\right] g_{\text {tot }}^{2}$} \\
\hline
\end{tabular}

\begin{tabular}{|ll|}
\hline & Covariant \\
\hline $16 \pi^{2} \beta_{\xi}$ & $-\frac{1}{4}(6 \xi-4) g_{\text {tot }}^{2}+2 \lambda$ \\
$16 \pi^{2} \beta_{\mu^{2}}$ & $-\frac{1}{2}\left[\mu^{2}-(8 \xi-6) \kappa^{2} V_{0}\right] g_{\text {tot }}^{2}+6\left[\mu^{2}+(4 \xi-2) \kappa^{2} V_{0}\right] \lambda$ \\
$16 \pi^{2}\left(\beta_{\mu^{2}}+4 \kappa^{2} V_{0} \beta_{\xi}\right)$ & {$\left[6 \mu_{E}^{2}-4 \kappa^{2} V_{0}\right] \lambda-\frac{1}{2}\left[\mu_{E}^{2}-2 \kappa^{2} V_{0}\right] g_{\text {tot }}^{2}$} \\
\hline
\end{tabular}

Table 3. $\mathrm{W}$ and $\mathrm{Z}$ vector boson contributions to the $\beta$-functions for the curvature coupling and the mass of a gravity coupled scalar field at leading order in $\kappa^{4} V_{0}$. The Jordan frame result has been calculated directly from the original action. The covariant result uses a geodesic expansion on field space and is independent of the frame used. The renormalisation group flow of $\mu_{E}^{2}=\mu^{2}+4 \kappa^{2} V_{0} \xi$ is the same for each of these approaches.

\begin{tabular}{|lll|}
\hline & Jordan frame & Covariant \\
\hline $16 \pi^{2} \beta_{\lambda}$ & $6 \lambda^{2}-\lambda g_{\text {tot }}^{2}+\frac{3}{8} \sum g^{4}$ & $6 \lambda^{2}-\lambda g_{\text {tot }}^{2}+\frac{3}{8} \sum g^{4}$ \\
$16 \pi^{2} \beta_{6}$ & $18 \lambda \lambda_{6}-\frac{3}{2} \lambda_{6} g_{\text {tot }}^{2}$ & $18 \lambda \lambda_{6}-\frac{3}{2} \lambda_{6} g_{\text {tot }}^{2}+3 \xi \lambda g_{\text {tot }}^{2}-\frac{9}{4} \lambda g_{\text {tot }}^{2}+9(2 \xi-1) \lambda^{2}$ \\
$16 \pi^{2} \gamma_{g}$ & $(6 \xi-1) \kappa^{2} \mu^{2}$ & $2 \kappa^{2} \mu_{E}^{2}-\frac{23}{2} \kappa^{4} V_{0}$ \\
\hline
\end{tabular}

Table 4. $\mathrm{W}$ and $\mathrm{Z}$ vector boson contributions to the $\beta$-functions for the quartic scalar self-coupling $\lambda$ and the sixth order scalar self-coupling $\lambda_{6}$ of a gravity coupled scalar field at at leading order in $\kappa^{4} V_{0}$. The metric wave function anomalous dimension is given to order $\kappa^{4} V_{0}$. The covariant result uses a geodesic expansion on field space and is independent of the frame used.

the three colours of the $\mathrm{SU}(3)$ gauge group. With such a simple operator it is best to use general results for the adiabatic expansion coefficients [30],

$$
b_{2}\left(-\nabla^{2}+E\right)=2\left(E-\frac{1}{6} R\right)^{2}+O\left(R^{2}\right)
$$

The wave-function renormalisation at one loop is $16 \pi^{2} \gamma_{\phi}=3 y^{2}$. The beta functions inferred from the renormalisation group equation (1.2) are,

$$
\begin{aligned}
16 \pi^{2} \beta_{\xi} & =(6 \xi-1) y^{2}, \\
16 \pi^{2} \beta_{\mu^{2}} & =6 y^{2} \mu^{2}, \\
16 \pi^{2} \beta_{\lambda} & =-6 y^{4}+12 \lambda y^{2} .
\end{aligned}
$$

There is no contribution to $\beta_{6}$ and $\gamma_{g}$ at one loop order. 


\section{The effective potential and stability}

We have argued that the most important contributions to the corrected Higgs potential are independent of the conformal frame and the stability analysis will be unambiguous as long as $\kappa^{4} V_{0}$ is negligible. To see how this works out in practice, we will consider instanton induced Higgs vacuum decay during inflation. Higgs instability is caused by negative values of the Higgs quartic coupling $\lambda_{\text {eff }}$ at large $\phi$. The Higgs vacuum is protected by a potential barrier, but the present Higgs vacuum cannot survive a period of inflation if quantum fluctuations take the Higgs field through the potential barrier. A large, positive, value for $\mu_{\text {eff }}^{2}$ is helpful because it reduces the vacuum tunnelling rate [4].

Perturbative fluctuations in the Higgs field on the scale of the horizon have a magnitude of order the expansion rate $H$. The vacuum will decay very rapidly if these fluctuations are larger than the barrier width. If we denote the value of the field at the maximum of the potential by $\phi_{b}$, then rapid vacuum decay occurs for $H>\phi_{b}$. If $H<\phi_{b}$, and $\left|V^{\prime \prime}\left(\phi_{b}\right)\right|<4 H^{2}$, then the main contribution to vacuum decay can be calculated using an instanton solution with the topology of a four-sphere and constant field $\phi=\phi_{b}$ [49]. The instanton induced tunnelling rate $\Gamma_{D}$ is given by

$$
\Gamma_{D}=A e^{-B}
$$

where the dominant effect is due to the exponent, given by the difference in action

$$
B=S_{E}\left[\phi_{b}\right]-S_{E}[0]
$$

The pre-factor $A$ should be very roughly of order $H^{4}$ on dimensional grounds, so that we can think of $e^{-B}$ as the decay rate per horizon volume per expansion time.

Consider a classical Higgs action which is given by the Euclidean Jordan frame Lagrangian,

$$
\mathcal{L}_{E}=\frac{1}{2}(\nabla \phi)^{2}+V(\phi)-\frac{1}{\kappa^{2}} U(\phi) R
$$

The Einstein equation (4.4) applied to the instanton four-sphere gives $U R=4 \kappa^{2} V$. The Lagrangian is constant, and we only need to multiply the Lagrangian by the volume of a four-sphere of radius $\sqrt{12 / R}$ to get the classical action,

$$
S_{E}[\phi]=-\frac{24 \pi^{2} U^{2}}{\kappa^{4} V} .
$$

The same result can be obtained from the Einstein frame action with potential $V_{E}=V / U^{2}$. If we now truncate the difference in action (7.2) to $O\left(\kappa^{0}\right)$, as in eqs. (3.2)-(3.4), then the contribution to the exponent is

$$
B=\frac{24 \pi^{2}}{9 H^{4}}\left[\frac{1}{2}\left(\mu^{2}+12 H^{2} \xi\right) \phi_{b}^{2}+\frac{1}{4} \lambda \phi_{b}^{4}\right] .
$$

$H$ is the vacuum expansion rate, $3 H^{2}=\kappa^{2} V_{0}$. The top of the potential barrier is determined by the scalar field equation eq. (4.5), which is equivalent to

$$
V_{E}^{\prime}\left(\phi_{b}\right)=0
$$


Note that all the main features of vacuum decay are determined by the Einstein frame potential even when we start out in the Jordan frame. This approach was used to calculate tunnelling rates in ref. [50].

We will assume that the quantum corrections are taken into account by replacing the classical action in the tunnelling exponent with the renormalisation group corrected effective action,

$$
B=\Gamma_{E}\left[\phi_{b}\right]-\Gamma_{E}[0]
$$

where $\Gamma_{E}$ uses the effective couplings. In particular,

$$
V_{\text {eff }}=\frac{1}{2} \mu_{\text {eff }}^{2}(\phi) \phi^{2}+\frac{1}{4} \lambda_{\text {eff }}(\phi) \phi^{4}
$$

where $\mu_{\text {eff }}^{2}$ is the combination $\mu_{\text {eff }}^{2}=\mu^{2}+12 \xi H^{2}$. The couplings are obtained by solving the renormalisation group equations (1.3).

Initial conditions for the running couplings are set at some chosen point. We take this point to be $\phi=170 \mathrm{GeV}$, close to the top quark mass. Combining the results from the tables of beta functions and replacing the vacuum energy by the expansion rate $H$ gives

$$
16 \pi^{2} \frac{d \mu_{\mathrm{eff}}^{2}}{d t}=12\left(\mu_{\mathrm{eff}}^{2}-2 H^{2}\right) \lambda-\frac{1}{2}\left(\mu_{\mathrm{eff}}^{2}-6 H^{2}\right) g_{\mathrm{tot}}^{2}+6\left(\mu_{\mathrm{eff}}^{2}-2 H^{2}\right) y^{2},
$$

The value of the Higgs coupling is known from experiments at energies less than $1 \mathrm{TeV}$. The best available values of the Higgs and top quark masses imply that $\lambda(170 \mathrm{GeV})=$ 0.12577 [1]. These experiments are essentially at zero vacuum energy $V_{0} \approx 0$, but since there is no dependence on the vacuum energy $V_{0}$ in $\beta_{\lambda}$, we can take the experimental values over to the early universe where $V_{0}$ is large.

The value of $\mu^{2}$ for the Higgs field as determined in the laboratory is negligible compared to the value of $\xi R$ in the inflationary universe, but here we have to take care because of subtleties in the properties of light fields in de Sitter space [51]. We already see a hint of this in the covariant beta-function which is large, of order $H^{2} / 3$. In the Euclidean approach to quantum field theory, the infra-red problems lead to a breakdown of perturbation theory for $\mu_{\text {eff }}^{2} \lesssim \lambda^{1 / 2} H^{2}$ [40], so our treatment will only be valid above this bound. Stochastic approximations imply that the light Higgs field develops a mass $\mu_{\mathrm{eff}}^{2} \approx 0.3534 \lambda^{1 / 2} H^{2}[35,37,40,52]$. If we assume $\mu^{2}(170 \mathrm{GeV}) \ll H^{2}$, then this sets a lower limit for de Sitter space of $\xi(170 \mathrm{GeV})>0.029 \lambda^{1 / 2}$. We can say nothing about curvature couplings smaller than this because the techniques required for dealing with loop corrections with smaller effective mass scales are quite different from the ones we use here $[51,53]$.

The effective couplings using (7.9) are plotted in figure 1. The standard model couplings $\lambda, g, g^{\prime}$ and $y$ have been evolved simultaneously using the two-loop flat space beta functions given in [4]. The value of the top quark mass $m_{t}$ sets the scale of the Yukawa coupling $y$ and this has a significant effect on the running of the Higgs self-coupling, and the value of the field where $\lambda$ vanishes. (The location of the point $\lambda=0$ is not fixed very accurately by the renormalisation group corrected potential, and other two loop effects ought to be included. We have corrected for this by raising $\lambda(170 \mathrm{GeV})$ by $0.2 \%$.) 

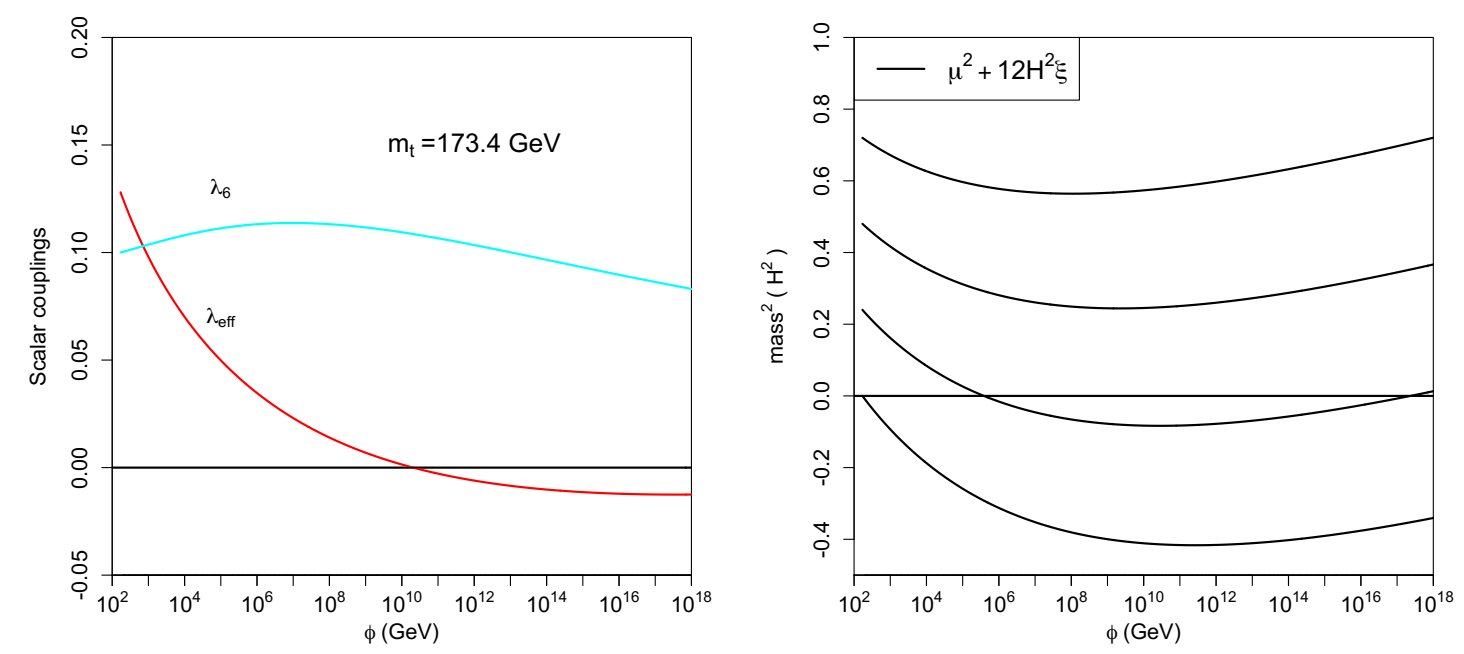

Figure 1. On the left, running couplings $\lambda$ and $\lambda_{6}$ with $m_{t}=173.4 \mathrm{GeV}$. On the right is the effective mass squared $\mu_{\text {eff }}^{2}=\mu^{2}+12 H^{2} \xi$. The initial conditions are $\lambda(170 \mathrm{GeV})=0.128$, $\lambda_{6}(170 \mathrm{GeV})=0.1$ and $\mu^{2}(170 \mathrm{GeV})=0$.

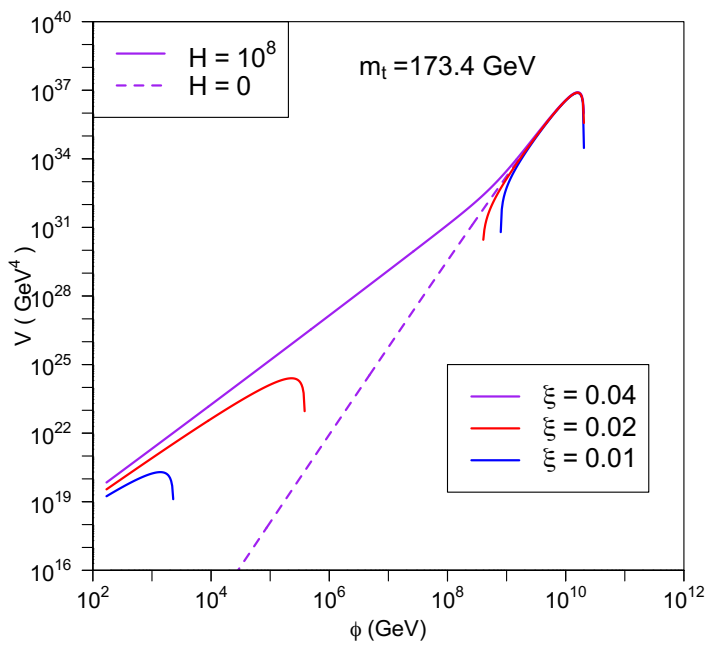

Figure 2. The effective Higgs potential plotted as a function of the Higgs field for $\mu^{2}=0$ and a range of curvature couplings $\xi$ at $170 \mathrm{GeV}$. There is a single maximum for large expansion rate $H$ or $\xi$, but new maxima and minima appear for small $H$ and $\xi$.

Note that for small initial values, the effective mass becomes negative at Higgs field values below the Planck scale. This can further drive the Higgs instability, and it can even give the potential a second maximum. This is illustrated by the potential plots in figure 2 . A combination of small initial $\mu_{\text {eff }}^{2}$ and small expansion rate $H$ leads to twin maxima.

The Higgs effective potential has been used to find the tunnelling exponents plotted in figure 3. The two plots show different values of the top quark mass, the first one closest in 

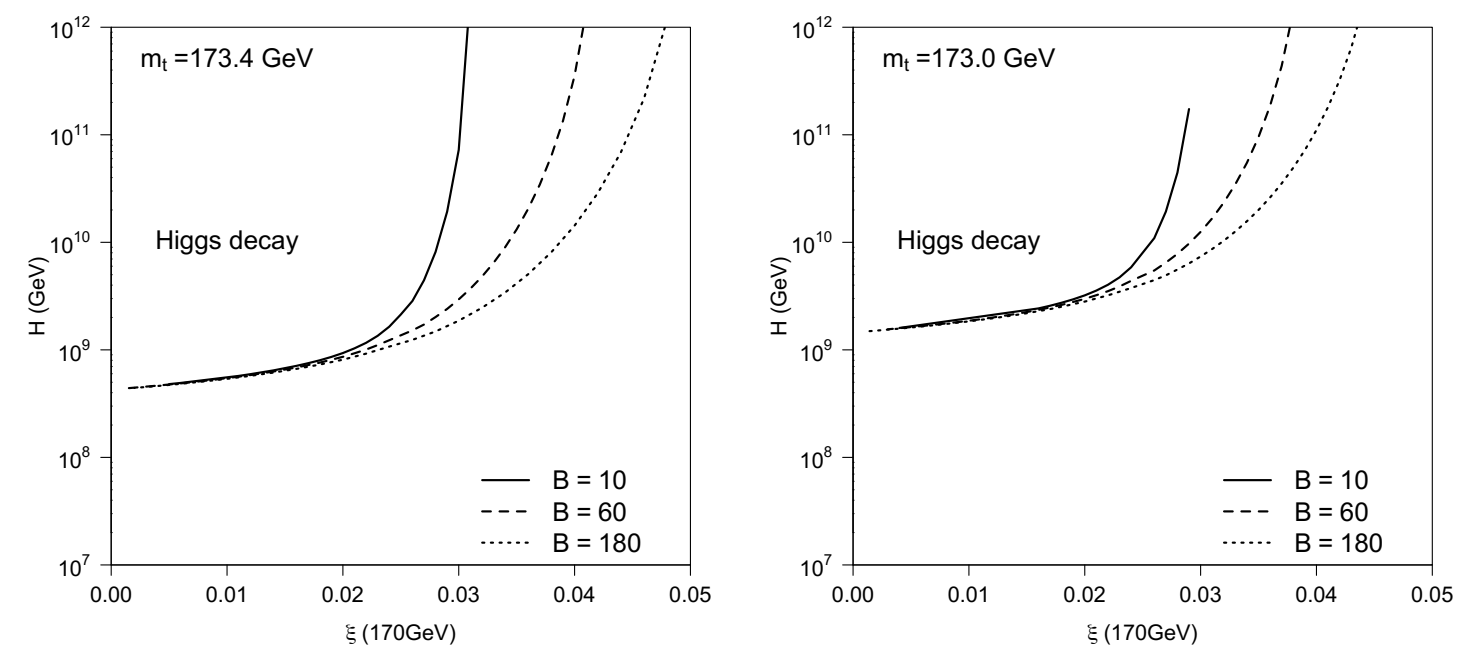

Figure 3. Higgs instability regions plotted as a function of the inflationary expansion rate and the curvature coupling $\xi$ at $170 \mathrm{GeV}$, assuming $\mu^{2}=0$ at $170 \mathrm{GeV}$. Lines show the exponent $B$, related to the vacuum decay rate per physical horizon volume, which is of order $e^{-B}$. The Higgs vacuum is unstable in the region above these curves.

line with the measured value and the second which favours Higgs stability. The results are plotted against the expansion rate of the Higgs vacuum $H$ and the value of the curvature coupling at $170 \mathrm{GeV}$, under the assumption that the Higgs mass square $\mu^{2}$ is negligible at small $\phi$. (This latter assumption is questionable, because only the combination $\mu_{\text {eff }}^{2}$ has a frame independent meaning when the non-covariant methodology is adopted.)

In the parameter region $\xi<0.022$ the potential has two maxima. Inside this region, the tunnelling rate is evaluated at the larger maximum. It is sometimes possible for the Higgs field to tunnel to the lower maximum, roll down the potential, and then tunnel up to the larger maximum. This combination is less likely than the single tunnelling event, and has no effect on the stability of the parameter region at the bottom of figure 3 . However, for small curvature coupling, the field could become trapped between the two maxima during inflation, and return to the present vacuum state after inflation.

The results support the idea first, proposed in ref. [4], that Higgs stability is sensitive to the value of the curvature coupling, though there are caveats concerning the case of negative $\xi$, as mentioned above. Running couplings were included in the Higgs stability analysis of [31]. The results are consistent with [31], although our results are more precise. Our main conclusion is that the stability analysis is independent of the choice of Jordan or Einstein frame.

Exactly how we interpret figure 3 is dependent on what we understand by the inflationary scenario. Suppose first of all that the universe is unique, with the minimum amount of inflation of around $N=60$ e-folds. The universe would then have started out one de Sitter horizon length across, and have grown to include $e^{3 N}$ de Sitter horizon regions at the end of inflation. The majority of these had to survive Higgs vacuum decay, leading to an extreme limit $B \gtrsim 180$. 
In the event that there are many universes like ours, then the conclusion is quite different because only one out of many universes has to survive Higgs vacuum decay. Espinosa et al. [4] argued that the survival probability of the universe can be calculated and should be $\propto \exp \left(-H^{2} N / 32 \phi_{b}^{2}\right)$. Assuming that the survival probability of our universe should be not be exponentially small gives a lower limit on the field at the top of the potential barrier $\phi_{b} \gtrsim H N^{1 / 2}$. This condition can be expressed in terms of $B$, for if we substitute this into eq. (7.5), then

$$
B \gtrsim \frac{\pi^{2}}{24} \frac{\mu_{\mathrm{eff}}^{2}}{H^{2}} N
$$

In practice $\mu_{\text {eff }}^{2} / H^{2} \sim 1$, and therefore $\phi_{b} \gtrsim H N^{1 / 2}$ corresponds very roughly to $B \gtrsim N$.

\section{Conclusion}

The Higgs-gravity system provides an interesting laboratory for trying out ideas in quantum gravity. One of the issues quantum gravity raises is how to define the spacetime geometry when scalar and metric backgrounds are allowed to mix freely together. We have found that applying a methodology which is fully covariant under such field redefinitions is perfectly feasible. This allows a complete physical equivalence between the different conformal frames. On the other hand, non-covariant approaches can still be used on scales below the Planck mass as long as we are careful. We advocate using the effective mass $\mu^{2}+\xi R$, since this has a covariant meaning, and the simpler Einstein frame can always be used. In particular, we have found that it is always possible to work consistently in a frame in which the curvature coupling vanishes. The dependence on curvature coupling in other frames can be recovered from relations like the beta function relations given in section 3 .

The effective action calculations we have done allow quite detailed results for Higgs vacuum decay which take into account running coupling constants, expanding on the work in [31]. As previously stated [4], the Higgs-curvature coupling can raise the potential barrier around the Higgs vacuum and stabilise the Higgs field during inflation. There are some caveats here, such as the extra metastable minima in the Higgs potential for small curvature coupling, and infra-red effects which set a lower bound to the effective Higgs mass, which deserve further study.

In one respect, the approach adopted has not been as general as it could, and maybe should, be. The covariant effective action has been used, but field redefinitions have not been fully integrated with the renormalisation group. In a fully general treatment, the renormalised field $\phi_{R}=Z\left(\mu_{R}\right) \phi$ should become a non-linear mapping into field space, $\phi_{R}=\phi_{R}\left(\phi, \mu_{R}\right)$. We have have not attempted this, in order to retain as much familiarity with conventional renormalisation group methods as possible.

Finally, we should point out that we have used existing non-covariant results for the standard model beta functions which are not associated with the spacetime curvature. This could cause problems if the running couplings depend on gauge parameters. In fact, the ' $g^{2} \lambda$ ' terms in $\beta_{\lambda}$ are dependent on gauge parameters [15]. The field value at which the quartic Higgs coupling become negative is not protected by any Nielsen identities and 
may well be gauge parameter dependent. We think that may be something to learn from gauge-parameter dependence for Higgs instability in flat space.

\section{Acknowledgments}

We would like to acknowledge useful discussions with Gerasimos Rigopoulos. IGM is supported by the Leverhulme Trust, grant RPG-2016-233 and receives some support from the Science and Facilities Council of the United Kingdom, grant number ST/P000371/1.

\section{A Mode decomposition matrices}

The differential operators for the gravity-Higgs system reduce to matrices when acting on the sphere in a tensor harmonic basis. The eigenvalues of the irreducible tensors are $\lambda_{T}$, $\lambda_{V}$ and $\lambda_{S}$ for tensor, vector and scalar harmonics respectively. The eigenvalues of the matrix $\Delta=\Pi\left(-\nabla^{2}+E\right) \Pi$ and the ghost matrix $Q$ are used to obtain zeta functions and beta functions. These matrices are given explicitly below.

The Laplacian

$$
\left(-\nabla^{2}\right)^{I}{ }_{J}=\left(\begin{array}{ccccc}
\lambda_{T} & 0 & 0 & 0 & 0 \\
0 & \lambda_{V}-\frac{5}{12} R & 0 & 0 & 0 \\
0 & 0 & \lambda_{S}-\frac{2}{3} R & 0 & 0 \\
0 & 0 & 0 & \lambda_{S} & 0 \\
0 & 0 & 0 & 0 & \lambda_{S}
\end{array}\right)
$$

The metric on field space,

$$
\mathcal{G}_{I J}=\left(\begin{array}{ccccc}
U & 0 & 0 & 0 & 0 \\
0 & 2 U\left(\lambda_{V}-\frac{1}{4} R\right) & 0 & 0 & 0 \\
0 & 0 & \frac{3}{4} U \lambda_{S}\left(\lambda_{S}-\frac{1}{3} R\right) & 0 & 0 \\
0 & 0 & 0 & -4 U & -2 \kappa U^{\prime} \\
0 & 0 & 0 & -2 \kappa U^{\prime} & K+\frac{1}{2} \kappa^{2} U^{\prime 2} / U
\end{array}\right) .
$$

The non-covariant mass matrix, writing $m_{T}^{2}=\frac{2}{3} U R-2 \kappa^{2} V$,

$$
E_{g I J}=\left(\begin{array}{ccccc}
m_{T}^{2} & 0 & 0 & 0 & 0 \\
0 & 2 m_{T}^{2}\left(\lambda_{V}-\frac{1}{4} R\right) & 0 & 0 & 0 \\
0 & 0 & \frac{3}{4} m_{T}^{2}\left(\lambda_{S}-\frac{1}{3} R\right) & 0 & 0 \\
0 & 0 & 0 & 8 \kappa^{2} V & 4 \kappa V^{\prime} \\
0 & 0 & 0 & 4 \kappa V^{\prime} & V^{\prime \prime}-\frac{1}{2 \kappa^{2}} R U^{\prime \prime}
\end{array}\right) .
$$

The covariant mass matrix including the connection terms

$$
E_{I J}=\left(\begin{array}{ccccc}
m_{T}^{2} & 0 & 0 & 0 & 0 \\
0 & 2 m_{T}^{2}\left(\lambda_{V}-\frac{1}{4} R\right) & 0 & 0 & 0 \\
0 & 0 & \frac{3}{4} m_{T}^{2}\left(\lambda_{S}-\frac{1}{3} R\right) & 0 & 0 \\
0 & 0 & 0 & 8 \kappa^{2} V & 2 \kappa V^{\prime}+\kappa^{-1} R U^{\prime} \\
0 & 0 & 0 & 2 \kappa V^{\prime}+\kappa^{-1} R U^{\prime} & M^{2}
\end{array}\right),
$$


where $M^{2}=V^{\prime \prime}-2 \kappa^{2} K V+\frac{1}{2} K U R-2 V U^{\prime \prime}$. The gauge transformation matrix

$$
\mathcal{R}_{\alpha}^{I}=\left(\begin{array}{cc}
0 & 0 \\
1 & 0 \\
0 & 2 \\
0 & \frac{1}{2} \lambda_{S} \\
0 & 0
\end{array}\right)
$$

The projection matrix $\Pi^{I}{ }_{J}=\delta^{I}{ }_{J}-\mathcal{R}^{I}{ }_{\alpha} \mathcal{N}^{\alpha \beta} \mathcal{R}_{J \beta}$,

$$
\Pi_{J}^{I}=\frac{1}{\lambda_{S}-\frac{1}{2} R}\left(\begin{array}{ccccc}
\lambda_{S}-\frac{1}{2} R & 0 & 0 & 0 & 0 \\
0 & 0 & 0 & 0 & 0 \\
0 & 0 & -\frac{1}{2} \lambda_{S} & -2 & 0 \\
0 & 0 & \frac{3}{8}\left(\lambda_{S}-\frac{1}{3} R\right) & \frac{3}{2}\left(\lambda_{S}-\frac{1}{3} R\right) & 0 \\
0 & 0 & 0 & 0 & \lambda_{S}-\frac{1}{2} R
\end{array}\right)
$$

The ghost operator

$$
Q^{\alpha}{ }_{\beta}=\mathcal{R}^{I \alpha} \mathcal{R}_{I \beta}=\left(\begin{array}{cc}
\lambda_{V}-\frac{1}{4} R & 0 \\
0 & \lambda_{S}-\frac{1}{2} R
\end{array}\right) .
$$

The ghost metric

$$
\gamma_{\alpha \beta}=\left(\begin{array}{cc}
2 U & 0 \\
0 & 2 U \lambda_{S}
\end{array}\right)
$$

\section{B Zeta-function evaluation}

Generalised zeta-functions for the operator eigenvalues on a four-sphere can be evaluated by using a standard binomial expansion method [46, 48]. Eigenvalues of the Laplacian are quadratic in $n$, but after diagonalisation of our operators some of the eigenvalues are non-polynomial, and a modification of the usual techniques is required.

We start with the Laplacian eigenvalues,

$$
\begin{aligned}
& \lambda_{S}=\frac{R}{12}\left[\left(n+\frac{3}{2}\right)^{2}-\frac{9}{4}\right] \\
& \lambda_{V}=\frac{R}{12}\left[\left(n+\frac{5}{2}\right)^{2}-\frac{13}{4}\right] \\
& \lambda_{T}=\frac{R}{12}\left[\left(n+\frac{7}{2}\right)^{2}-\frac{17}{4}\right]
\end{aligned}
$$


The degeneracies of these eigenvalues are

$$
\begin{aligned}
& g_{S}=\frac{1}{3}\left(n+\frac{3}{2}\right)^{3}-\frac{1}{12}\left(n+\frac{3}{2}\right) \\
& g_{V}=\left(n+\frac{5}{2}\right)^{3}-\frac{9}{4}\left(n+\frac{5}{2}\right) \\
& g_{T}=\frac{5}{3}\left(n+\frac{7}{2}\right)^{3}-\frac{125}{12}\left(n+\frac{7}{2}\right)
\end{aligned}
$$

The eigenvalues $\lambda_{n}$ obtained after diagonalisation are algebraic functions of these eigenvalues which can be expanded for large $n$ as power series

$$
\lambda_{n}=\frac{1}{12} R\left[(n+a)^{2}+A+B(n+a)^{-2}+\ldots\right] .
$$

The degeneracies are generally

$$
g_{n}=b(n+a)^{3}+c(n+a) .
$$

We replace $\lambda_{n}^{-s}$ in the zeta-function by its binomial expansion, and then sums of powers of $n+a$ can be replaced with Hurwitz zeta-functions $\zeta_{H}(s, a)$,

$$
\zeta_{H}(s, a)=\sum_{n=0}^{\infty}(n+a)^{-s} .
$$

The Hurwitz zeta-functions have an analytic extension with a pole at $s=0$ with residue 1. Values at $s=-1$ and $s=-3$ are Bernoulli polynomials,

$$
\zeta(-1, a)=-\frac{1}{2} B_{2}(a), \quad \zeta(-3, a)=-\frac{1}{4} B_{4}(a)
$$

After rearranging the summations we arrive at an expression for the zeta function,

$$
\zeta(s)=\left(\frac{R}{12}\right)^{-s}\{b f(s)+c g(s)\}
$$

where

$$
\begin{aligned}
& f(s)=\zeta_{H}(2 s-3, a)-s B \zeta_{H}(2 s+1, a)+\frac{1}{2} s(s+1) A^{2} \zeta_{H}(2 s+1, a)+\ldots \\
& g(s)=\zeta_{H}(2 s-1, a)-s A \zeta_{H}(2 s+1, a)+\ldots
\end{aligned}
$$

All of the terms denoted by $\ldots$ vanish at $s=0$ and we are left with

$$
\zeta(0)=-\frac{1}{2} c B_{2}(a)-\frac{1}{4} b B_{4}(a)-\frac{c}{2} A-\frac{b}{4}\left(2 B-A^{2}\right) .
$$

The zeta-function sum is always taken from $n=0$, but some of the derived modes are identically zero for some $n$. For example, the gradient of a constant scalar mode does not 
give a valid vector mode. These exceptions are handled by subtracting the contributions from the $N(h)$ non-existent modes,

$$
\zeta(0)=\left(\sum_{n=0}^{\infty} g_{n} \lambda_{n}^{-s}\right)_{s=0}-N(h)
$$

In particular,

$$
N\left(\nabla_{\mu} h^{S}\right)=1, \quad N\left(\nabla_{(\mu} h_{\nu)}^{V}\right)=10, \quad N\left(\nabla_{\mu \nu} h^{S}\right)=6,
$$

the last factor consisting of one $n=0$ and five $n=1$ scalar modes with vanishing second derivative.

Open Access. This article is distributed under the terms of the Creative Commons Attribution License (CC-BY 4.0), which permits any use, distribution and reproduction in any medium, provided the original author(s) and source are credited.

\section{References}

[1] G. Degrassi et al., Higgs mass and vacuum stability in the Standard Model at NNLO, JHEP 08 (2012) 098 [arXiv: 1205.6497] [INSPIRE].

[2] D. Buttazzo et al., Investigating the near-criticality of the Higgs boson, JHEP 12 (2013) 089 [arXiv: 1307.3536] [INSPIRE].

[3] K. Blum, R. T. D'Agnolo and J. Fan, Vacuum stability bounds on Higgs coupling deviations in the absence of new bosons, JHEP 03 (2015) 166 [arXiv:1502.01045] [INSPIRE].

[4] J.R. Espinosa, G.F. Giudice and A. Riotto, Cosmological implications of the Higgs mass measurement, JCAP 05 (2008) 002 [arXiv:0710.2484] [INSPIRE].

[5] C.P. Burgess, Quantum gravity in everyday life: General relativity as an effective field theory, Living Rev. Rel. 7 (2004) 5 [gr-qc/0311082] [INSPIRE].

[6] D.P. George, S. Mooij and M. Postma, Quantum corrections in Higgs inflation: the real scalar case, JCAP 02 (2014) 024 [arXiv:1310.2157] [INSPIRE].

[7] C.F. Steinwachs and A. Yu. Kamenshchik, Non-minimal Higgs Inflation and Frame Dependence in Cosmology, arXiv:1301.5543 [INSPIRE].

[8] A. Yu. Kamenshchik and C.F. Steinwachs, Question of quantum equivalence between Jordan frame and Einstein frame, Phys. Rev. D 91 (2015) 084033 [arXiv:1408.5769] [INSPIRE].

[9] D.P. George, S. Mooij and M. Postma, Quantum corrections in Higgs inflation: the Standard Model case, JCAP 04 (2016) 006 [arXiv: 1508.04660] [InSPIRE].

[10] I.G. Moss, Covariant one-loop quantum gravity and Higgs inflation, arXiv:1409.2108 [INSPIRE].

[11] I.G. Moss, Vacuum stability and the scaling behaviour of the Higgs-curvature coupling, arXiv: 1509.03554 [INSPIRE].

[12] G.A. Vilkovisky, The gospel according to dewitt, in Quantum Theory of Gravity, Adam Hilger, London U.K. (1984), pg. 169.

[13] B.S. DeWitt, Dynamical Theory of Groups and Fields, Gordon and Breach, Philadelphia U.S.A. (1965). 
[14] A.O. Barvinsky and G.A. Vilkovisky, The generalized Schwinger-Dewitt technique in gauge theories and quantum gravity, Phys. Rept. 119 (1985) 1 [INSPIRE].

[15] L.E. Parker and D.J. Toms, Quantum Field Theory in Curved Spacetime, Cambridge University Press, Cambridge U.K. (2009).

[16] L. Alvarez-Gaumé, D.Z. Freedman and S. Mukhi, The background field method and the ultraviolet structure of the supersymmetric nonlinear $\sigma$-model, Annals Phys. 134 (1981) 85.

[17] P.S. Howe, G. Papadopoulos and K.S. Stelle, The Background Field Method and the Nonlinear $\sigma$ Model, Nucl. Phys. B 296 (1988) 26 [INSPIRE].

[18] N. Nielsen, On the gauge dependence of spontaneous symmetry breaking in gauge theories, Nucl. Phys. B 101 (1975) 173.

[19] R. Fukuda and T. Kugo, Gauge Invariance in the Effective Action and Potential, Phys. Rev. D 13 (1976) 3469 [INSPIRE].

[20] R. Kobes, G. Kunstatter and A. Rebhan, Gauge dependence identities and their application at finite temperature, Nucl. Phys. B 355 (1991) 1 [INSPIRE].

[21] C. Contreras and L. Vergara, The Nielsen identities for the generalized R(epsilon) gauge, Phys. Rev. D 55 (1997) 5241 [Erratum ibid. D 56 (1997) 6714] [hep-th/9610109] [InSPIRE].

[22] T. Markkanen, S. Nurmi and A. Rajantie, Do metric fluctuations affect the Higgs dynamics during inflation?, JCAP 12 (2017) 026 [arXiv:1707.00866] [INSPIRE].

[23] C.P. Burgess and G. Kunstatter, On the Physical Interpretation of the Vilkovisky-de Witt Effective Action, Mod. Phys. Lett. A 2 (1987) 875 [Erratum ibid. A 2 (1987) 1003] [InSPIRE].

[24] B.S. DeWitt, The effective action, in Quantum Field Theory and Quantum Statistics. Vol. 1, Adam Hilger, London U.K. (1987), pg. 191.

[25] I. Krive and A.D. Linde, On the Vacuum stability problem in gauge theories, Nucl. Phys. B 117 (1976) 265.

[26] H.D. Politzer and S. Wolfram, Bounds on Particle Masses in the Weinberg-Salam Model, Phys. Lett. 82B (1979) 242 [Erratum ibid. B 83 (1979) 421] [INSPIRE].

[27] N. Cabibbo, L. Maiani, G. Parisi and R. Petronzio, Bounds on the Fermions and Higgs Boson Masses in Grand Unified Theories, Nucl. Phys. B 158 (1979) 295 [inSPIRE].

[28] M. Sher, Electroweak Higgs Potentials and Vacuum Stability, Phys. Rept. 179 (1989) 273 [INSPIRE].

[29] S.R. Coleman and E.J. Weinberg, Radiative Corrections as the Origin of Spontaneous Symmetry Breaking, Phys. Rev. D 7 (1973) 1888 [INSPIRE].

[30] D.V. Vassilevich, Heat kernel expansion: User's manual, Phys. Rept. 388 (2003) 279 [hep-th/0306138] [INSPIRE].

[31] M. Herranen, T. Markkanen, S. Nurmi and A. Rajantie, Spacetime curvature and the Higgs stability during inflation, Phys. Rev. Lett. 113 (2014) 211102 [arXiv:1407.3141] [INSPIRE].

[32] A.D. Plascencia and C. Tamarit, Convexity, gauge-dependence and tunneling rates, JHEP 10 (2016) 099 [arXiv: 1510.07613] [INSPIRE].

[33] M. Endo, T. Moroi, M.M. Nojiri and Y. Shoji, On the Gauge Invariance of the Decay Rate of False Vacuum, Phys. Lett. B 771 (2017) 281 [arXiv:1703.09304] [INSPIRE]. 
[34] A. Rajantie and S. Stopyra, Standard Model vacuum decay in a de Sitter Background, Phys. Rev. D 97 (2018) 025012 [arXiv: 1707.09175] [InSPIRE].

[35] A.A. Starobinsky and J. Yokoyama, Equilibrium state of a selfinteracting scalar field in the de Sitter background, Phys. Rev. D 50 (1994) 6357 [astro-ph/9407016] [INSPIRE].

[36] C.P. Burgess, L. Leblond, R. Holman and S. Shandera, Super-Hubble de Sitter Fluctuations and the Dynamical RG, JCAP 03 (2010) 033 [arXiv:0912.1608] [INSPIRE].

[37] B. Garbrecht and G. Rigopoulos, Self Regulation of Infrared Correlations for Massless Scalar Fields during Inflation, Phys. Rev. D 84 (2011) 063516 [arXiv: 1105.0418] [INSPIRE].

[38] J. Serreau, Effective potential for quantum scalar fields on a de Sitter geometry, Phys. Rev. Lett. 107 (2011) 191103 [arXiv: 1105.4539] [INSPIRE].

[39] A. Rajaraman, On the proper treatment of massless fields in Euclidean de Sitter space, Phys. Rev. D 82 (2010) 123522 [arXiv: 1008.1271] [INSPIRE].

[40] M. Beneke and P. Moch, On "dynamical mass" generation in Euclidean de Sitter space, Phys. Rev. D 87 (2013) 064018 [arXiv:1212.3058] [INSPIRE].

[41] I.G. Moss and D.J. Toms, Invariants of the heat equation for non-minimal operators, J. Phys. A 47 (2014) 215401 [arXiv:1311.5445] [InSPIRE].

[42] I.L. Buchbinder and S.D. Odintsov, Effective Potential and Phase Transitions Induced by Curvature in Gauge Theories in Curved Space-time, Yad. Fiz. 42 (1985) 1268 [Class. Quant. Grav. 2 (1985) 721] [INSPIRE].

[43] A.O. Barvinsky, A. Yu. Kamenshchik, C. Kiefer, A.A. Starobinsky and C.F. Steinwachs, Higgs boson, renormalization group and naturalness in cosmology, Eur. Phys. J. C 72 (2012) 2219 [arXiv: 0910.1041] [INSPIRE].

[44] A.O. Barvinsky, A. Yu. Kamenshchik, C. Kiefer, A.A. Starobinsky and C. Steinwachs, Asymptotic freedom in inflationary cosmology with a non-minimally coupled Higgs field, JCAP 12 (2009) 003 [arXiv:0904.1698] [INSPIRE].

[45] A.O. Barvinsky, Standard Model Higgs Inflation: CMB, Higgs Mass and Quantum Cosmology, Prog. Theor. Phys. Suppl. 190 (2011) 1 [arXiv:1012.4523] [INSPIRE].

[46] J.S. Dowker, Effective action in spherical domains, Commun. Math. Phys. 162 (1994) 633 [hep-th/9306154] [INSPIRE].

[47] G. Cognola, E. Elizalde, S. Nojiri, S.D. Odintsov and S. Zerbini, One-loop $f(R)$ gravity in de Sitter universe, JCAP 02 (2005) 010 [hep-th/0501096] [INSPIRE].

[48] E. Elizalde, S.D. Odintsov, A. Romeo, A.A. Bytsenko and S. Zerbini, Zeta regularization techniques with applications, World Scientific, Singapore (1994).

[49] S.W. Hawking and I.G. Moss, Supercooled Phase Transitions in the Very Early Universe, Phys. Lett. 110B (1982) 35 [INSPIRE].

[50] X. Calmet, I. Kuntz and I.G. Moss, Non-Minimal Coupling of the Higgs Boson to Curvature in an Inflationary Universe, Found. Phys. 48 (2018) 110 [arXiv:1701.02140] [InSPIRE].

[51] S. Weinberg, Quantum contributions to cosmological correlations, Phys. Rev. D 72 (2005) 043514 [hep-th/0506236] [INSPIRE].

[52] I. Moss and G. Rigopoulos, Effective long wavelength scalar dynamics in de Sitter, JCAP 05 (2017) 009 [arXiv: 1611.07589] [INSPIRE]. 
[53] E.O. Kahya and R.P. Woodard, Quantum Gravity Corrections to the One Loop Scalar Self-Mass during Inflation, Phys. Rev. D 76 (2007) 124005 [arXiv:0709. 0536] [INSPIRE]. 